\title{
PROJET LANGEVIN-WALLON
}

\section{SOMMAIRE}

$1^{\circ}$ Introduction ;

$2^{\circ}$ Structure et organisation de l'enseignement ;

$3^{\circ}$ Formation des maîtres ;

$4^{\circ}$ Organes de contrôle et de perfectionnement ;

$5^{\circ}$ Programmes, Horaires, Méthodes, Sanction des études ;

$6^{\circ}$ Education morale et civique ; formation de l'homme et du citoyen ;

$7^{\circ}$ Education populaire ;

$8^{\circ}$ Délais d'application.

Appendice

a) Projet de l'organisation de l'enseignement dans les régions rurales ;

b) Voeux émis par la Commission au cours de ses séances.

\section{Composition de la Commission}

Présidents :

MM.

LANGEVIN (Paul), professeur au Collège de France, président du Groupe français d'Education nouvelle (1944-Déc.1946).

WALLON (Henri), professeur au Collège de France, président de la Société française de Pédagogie.

Vice-président :

M. PIERON (Henri), professeur au Collège de France, directeur de l'Institut d'orientation professionnelle.

Membres :

Mlle CABANE, inspectrice primaire ; 
MM.

CANONGE, professeur de collège technique ;

COORMAERT, professeur au Collège de France ;

DURRY, professeur à la Sorbonne ;

FEBVRE (Lucien), professeur au Collège de France ;

GAL, professeur agrégé, attaché à la Direction de l'Enseignement du Second degré ;

GEORGE (Pierre), professeur agrégé à Paris ;

GRANDJOUAN, chef du Service de Documentation et d'Etudes pédagogiques au Ministère ;

JANETS, professeur agrégé à Paris ;

LAVERGNE, professeur de Collège moderne à Paris ;

PARODI, inspecteur général honoraire de l'instruction publique ;

RENAUDEAU, directeur de l'Ecole normale supérieure d'Enseignement technique ;

Madame SECLET, professeur à l'Ecole normale d'institutrices de Paris.

MM.

SENEZE, instituteur à Paris ;

TEISSIER, professeur à la Sorbonne ;

VOGUET, instituteur à Paris ;

WEILLER, professeur au lycée Henri IV, attaché à la Direction de l'Enseignement du Second degré.

MM.

BAYET, au titre de Directeur Général de l'Enseignement ;

AUGER, au titre de Directeur de l'Enseignement Supérieur ;

MONOD, au titre de Directeur de l'Enseignement du Second Degré ;

LE ROLLAND, au titre de Directeur de l'Enseignement Technique ;

BARREE, au titre de Directeur de l'Enseignement du Premier Degré ;

CORNU et BESLAIS, représentant le Ministre de l'Education Nationale,

ont également pris part aux travaux de la Commission. 


\section{INTRODUCTION}

L'enseignement français est depuis longtemps réputé dans le Monde pour sa haute qualité et sa valeur culturelle. Chaque année nos universités accueillent un très grand nombre d'étudiants étrangers. Notre enseignement secondaire jouit d'un semblable prestige. Enfin l'enseignement primaire, organisé par Jules Ferry, fut l'une des grandes oeuvres de la IIIe République. Il se propose de donner à tout homme, aussi humble que soit son origine, le minimum de connaissances indispensables pour faire de lui un citoyen conscient, pour enrichir son esprit et élargir son horizon. Enfin l'enseignement technique, organisé après la guerre 1914-18, a connu un rapide développement.

Cependant, dès avant cette guerre, il était devenu évident que notre enseignement devait être réorganisé. Des tentatives partielles de réforme avaient été esquissées. Pendant l'occupation, divers projets furent élaborés, tant à Alger que par les Résistants de la Métropole. Aujourd'hui une réforme complète est nécessaire et urgente : l'organisation actuelle de notre enseignement suscite en effet des critiques justifiées et ne lui permet pas d'assumer pleinement le rôle qui devrait être le sien dans une démocratie moderne. Tout d'abord cette organisation ne répond pas à un plan d'ensemble clairement conçu et exactement mis en oeuvre. Elle résulte d'un passé historique au cours duquel des institutions diverses furent créées sans être coordonnées. A des législations anciennes, d'autres, ajoutées et non substituées. De sorte que certains organismes se concurrencent inutilement ou font double emploi, cependant qu'il existe des lacunes et que l'enseignement ne répond pas à tous les besoins actuels. Le moment semble venu de procéder à une réorganisation générale pour remplacer cette construction disparate par un ensemble clairement ordonné et susceptible de satisfaire tous les besoins.

La structure de l'enseignement doit en effet être adaptée à la structure sociale. Depuis un demisiècle la structure de l'enseignement n'a pas été profondément modifiée. La structure sociale au contraire a subi une évolution rapide et des transformations fondamentales. Le machinisme, l'utilisation des sources nouvelles d'énergie, le développement des moyens de transport et de transmission, la concentration industrielle, l'accroissement de la production, l'entrée massive des femmes dans la vie économique, la diffusion de l'instruction élémentaire ont profondément modifié les conditions de vie et l'organisation sociale. La rapidité et l'ampleur du progrès économique, qui avaient rendu nécessaire en 1880 la diffusion de l'enseignement élémentaire dans les masses ouvrières, pose à présent le problème du recrutement d'un personnel de plus en plus nombreux de cadres et de techniciens. La bourgeoisie, héréditairement appelée à tenir les postes de direction et de responsabilité ne saurait plus désormais, seule, y suffire. Les besoins nouveaux de l'économie moderne posent la nécessité d'une refonte de notre enseignement qui, dans sa structure actuelle, n'est plus adapté aux conditions économiques et sociales.

Cette inadaptation de l'enseignement à l'état présent de la société a pour signe visible l'absence ou l'insuffisance des contacts entre l'école à tous les degrés et la vie. Les études primaires, secondaires, supérieures sont trop souvent en marge du réel. L'école semble un milieu clos, imperméable aux expériences du monde. Le divorce entre l'enseignement scolaire et la vie s'accentue par la permanence de nos institutions scolaires au sein d'une société en voie 
d'évolution accélérée. Ce divorce dépouille l'enseignement de son caractère éducatif. Une réforme est urgente qui remédiera à cette carence de l'enseignement dans l'éducation du producteur et du citoyen et lui permettra de donner à tous une formation civique, sociale, humaine.

Demeuré en marge de la vie, l'enseignement n'a pas tiré profit du progrès scientifique. L'empirisme et la tradition commandent ses méthodes alors qu'une pédagogie nouvelle, fondée sur les sciences de l'éducation, devrait inspirer et renouveler ses pratiques.

D'autre part, et à tous les degrés, l'enseignement méconnaît dans l'élève le futur citoyen. Il ne donne pas une importance suffisante à l'explication objective et scientifique des faits économiques et sociaux, à la culture méthodique de l'esprit critique, à l'apprentissage actif de l'énergie, de la liberté, de la responsabilité. Or, cette formation civique de la jeunesse est l'un des devoirs fondamentaux d'un état démocratique et c'est à l'enseignement public qu'il appartient de remplir ce devoir.

Toutes ces raisons justifient la nécessité d'une réforme profonde de nos institutions d'enseignement qui si elles ont dans le passé rempli avec succès leur mission doivent, pour rester à la hauteur d'une réputation méritée, se transformer et s'adapter à l'état économique et social actuel.

\section{PRINCIPES GENERAUX}

La reconstruction complète de notre enseignement repose sur un petit nombre de principes dont toutes les mesures envisagées dans l'immédiat ou à plus longue échéance seront l'application.

Le premier principe, celui qui par sa valeur propre et l'ampleur de ses conséquences domine tous les autres est le principe de justice. Il offre deux aspects non point opposés mais complémentaires : l'égalité et la diversité. Tous les enfants, quelles que soient leurs origines familiales, sociales, ethniques, ont un droit égal au développement maximum que leur personnalité comporte. Ils ne doivent trouver d'autre limitation que celle de leurs aptitudes. L'enseignement doit donc offrir à tous d'égales possibilités de développement, ouvrir à tous l'accès à la culture, se démocratiser moins par une sélection qui éloigne du peuple les plus doués que par une élévation continue du niveau culturel de l'ensemble de la nation. L'introduction de "la justice à l'école" par la démocratisation de l'enseignement mettra chacun à la place que lui assignent ses aptitudes, pour le plus grand bien de tous. La diversification des fonctions sera commandée non plus par la fortune ou la classe sociale mais par la capacité à remplir la fonction. La démocratisation de l'enseignement, conforme à la justice, assure une meilleure distribution des tâches sociales. Elle sert l'intérêt collectif en même temps que le bonheur individuel.

L'organisation actuelle de notre enseignement entretient dans notre société le préjugé antique d'une hiérarchie entre les tâches et les travailleurs. Le travail manuel, l'intelligence pratique sont encore trop souvent considérés comme de médiocre valeur. L'équité exige la reconnaissance de l'égale dignité de toutes les tâches sociales, de la haute valeur matérielle et morale des activités manuelles, de l'intelligence pratique, de la valeur technique. Ce reclassement des valeurs réelles est indispensable dans une société démocratique moderne dont le progrès et la vie même sont subordonnés à l'exacte utilisation des compétences. 
La réforme de notre enseignement doit être l'affirmation dans nos institutions du droit des jeunes à un développement complet. La législation d'une république démocratique se doit de proclamer et de protéger les droits des faibles, elle se doit de proclamer et de protéger le droit de tous les enfants, de tous les adolescents, à l'éducation. Celle-ci prendra pour base la connaissance de la psychologie des jeunes, l'étude objective de chaque individualité. Elle se fera dans le respect de la personnalité enfantine, afin de dégager et de développer en chacun les aptitudes originales. Le droit des jeunes à un développement complet implique la réalisation des conditions hygiéniques et éducatives les plus favorables. En particulier l'effectif des classes devra être tel que le maître puisse utilement s'occuper de chaque élève : il ne devra en aucun cas dépasser 25.

La mise en valeur des aptitudes individuelles en vue d'une utilisation plus exacte des compétences pose le principe de l'orientation. Orientation scolaire d'abord, puis orientation professionnelle doivent aboutir à mettre chaque travailleur, chaque citoyen au poste le mieux adapté à ses possibilités, le plus favorable à son rendement. A la sélection actuelle qui aboutit à détourner les plus doués de professions où ils pourraient rendre d'éminents services, doit se substituer un classement des travailleurs, fondé à la fois sur les aptitudes individuelles et les besoins sociaux.

C'est dire que l'enseignement doit comporter une part de culture spécialisée de plus en plus large à mesure que les aptitudes se dégagent et s'affirment. Mais la formation du travailleur ne doit en aucun cas nuire à la formation de l'homme. Elle doit apparaître comme une spécialisation complémentaire d'un large développement humain. "Nous concevons la culture générale, dit Paul Langevin, comme une initiation aux diverses formes de l'activité humaine, non seulement pour déterminer les aptitudes de l'individu, lui permettre de choisir à bon escient avant de s'engager dans une profession, mais aussi pour lui permettre de rester en liaison avec les autres hommes, de comprendre l'intérêt et d'apprécier les résultats d'activités autres que la sienne propre, de bien situer celle-ci par rapport à l'ensemble."

La culture générale représente ce qui rapproche et unit les hommes tandis que la profession représente trop souvent ce qui les sépare. Une culture générale solide doit donc servir de base à la spécialisation professionnelle et se poursuivre pendant l'apprentissage de telle sorte que la formation de l'homme ne soit pas limitée et entravée par celle du technicien. Dans un état démocratique, où tout travailleur est citoyen, il est indispensable que la spécialisation ne soit pas un obstacle à la compréhension de plus vastes problèmes et qu'une large et solide culture libère l'homme des étroites limitations du technicien.

C'est pourquoi le rôle de l'école ne doit pas se borner à éveiller le goût de la culture pendant la période de la scolarité obligatoire, quelle qu'en soit la durée. L'organisation nouvelle de l'enseignement doit permettre le perfectionnement continu du citoyen et du travailleur. En tout lieu, des immenses agglomérations urbaines jusqu'aux plus petits hameaux, l'école doit être un centre de diffusion de la culture. Par une adaptation exacte aux conditions régionales et aux besoins locaux, elle doit permettre à tous le perfectionnement de la culture. Dépositaire de la pensée, de l'art, de la civilisation passée, elle doit les transmettre en même temps qu'elle est l'agent actif du progrès et de la modernisation. Elle doit être le point de rencontre, l'élément de cohésion qui assure la continuité du passé et de l'avenir. 


\section{CONSEQUENCES DE CES PRINCIPES}

La première conséquence des principes qui viennent d'être énoncés est la reconstruction complète de notre enseignement sur un plan nouveau, présentant plus de simplicité, d'unité et de cohérence : la structure même de l'institution doit être modifiée pour répondre au principe de justice fondamental dans une démocratie.

Dans l'organisation actuelle, les divers ordres d'enseignement ne répondent pas toujours à leur but qui devrait être le développement maximum de tous ceux qui sont aptes à en profiter, mais de ceux-là seuls. Le recrutement en est aujourd'hui trop souvent déterminé par la classe sociale et les ressources financières des familles. Le passage d'un ordre d'enseignement à l'autre (primaire, secondaire, technique) présente de grandes difficultés. Aussi la proportion des enfants de familles ouvrières qui accèdent à l'enseignement supérieur et aux grandes écoles est infime.

La logique et l'équité exigent que les divers échelons de l'enseignement répondent à des niveaux de développement, puis à des spécialisations d'aptitudes et non à des catégories sociales. C'est pourquoi, dans la structure nouvelle, l'enseignement présentera des degrés progressifs correspondant à des niveaux de développement et auxquels tous les enfants devront successivement accéder.

L'enseignement du $1^{\text {er }}$ degré sera obligatoire pour tous les enfants de 11 à 18 ans et comprendra trois cycles successifs. Le $1^{\text {er }}$ cycle concernera tous les enfants de 3 à 11 ans, l'âge de l'obligation scolaire demeurant fixé à 6 ans. Pendant cette période, tous les enfants recevront un enseignement adapté à leur développement mental et susceptible de répondre à leurs besoins immédiats. Le rôle principal du $1^{\mathrm{er}}$ cycle sera de mettre l'enfant en possession des techniques de base qui lui permettront de comprendre et de se faire comprendre, l'étude du milieu physique et humain lui permettant de se situer dans l'espace et dans le temps.

A la fin de cette première étape, tous les enfants entreront obligatoirement dans les établissements du $2^{\mathrm{e}}$ cycle puis du $3^{\mathrm{e}}$ cycle. Ils y resteront jusqu'à 18 ans. L'obligation scolaire devra donc être prolongée de 4 années. Le $2^{\mathrm{e}}$ cycle (de 11 à 15 ans) sera une période d'orientation. Tout en assurant l'acquisition d'un complément indispensable de connaissances générales, il sera consacré à une observation méthodique des enfants pour déceler leurs aptitudes et permettre leur orientation. Le $3^{\mathrm{e}}$ cycle (de 15 à 18 ans) sera la période de détermination, il sera consacré à la formation du citoyen et du travailleur. Les élèves aptes à recevoir l'enseignement universitaire bénéficieront d'une formation théorique adaptée. Pour les autres, la culture générale se poursuivra en rapport avec une culture spécialisée orientée vers la profession, de sorte que les jeunes gens qui seraient désignés par leurs aptitudes pour l'exercice d'un métier seront aptes à la fin du 3 e cycle à entrer dans la vie professionnelle. L'enseignement du $3^{\mathrm{e}}$ cycle, en raison du rôle fondamental qu'il est appelé à jouer dans l'affectation sociale et l'utilisation des aptitudes, devra présenter une grande souplesse, une grande diversité, afin d'offrir des combinaisons d'études, des groupements de disciplines adaptés aux diverses catégories d'esprits. 
A la fin du $1^{\text {er }}$ degré et au-delà de la limite de l'obligation scolaire (18 ans), un second degré d'enseignement s'ouvrira à ceux, et à ceux-là seulement, qui se seront révélés capables d'en profiter. Elargi et diversifié, il orientera les étudiants vers des spécialisations de plus en plus définies, dans toutes les catégories d'aptitudes et d'activités. Les études supérieures techniques y trouveront place au même titre que les études littéraires, scientifiques et artistiques. L'un des rôles importants de cet enseignement supérieur sera d'assurer la formation des maîtres à tous les degrés. Des instituts hautement spécialisés s'ouvriront ensuite aux chercheurs de toutes catégories.

La seconde conséquence importante des principes directeurs $d$ a la réforme de l'enseignement est la nécessité de prévoir tout un ensemble de mesures de justice sociale, dont l'absence serait la négation de toute réforme.

En premier lieu, l'enseignement public doit être gratuit à tous les degrés. La gratuité s'impose comme le complément logique de l'obligation scolaire. La prolongation de la scolarité obligatoire jusqu'à 18 ans entraîne la gratuité de l'enseignement aux 3 cycles du $1^{\mathrm{er}}$ degré. Mais il est indispensable, si chacun doit occuper la place où il est susceptible de rendre le plus de services, que le recrutement de l'enseignement supérieur soit déterminé par les aptitudes et non par le rang social ou le niveau de fortune. Pour s'ouvrir à tous ceux qui en peuvent bénéficier, l'enseignement supérieur doit, lui aussi, être gratuit.

La gratuité inscrite dans les textes serait un leurre si on la limitait à la suppression des frais d'études sans s'inquiéter des conditions et des moyens de vie des élèves et des étudiants. Dans les familles ouvrières, le gain de l'adolescent est escompté. L'apprenti, le jeune ouvrier doivent apporter leur contribution au budget familial, et de cette contribution il est matériellement impossible de se passer. La gratuité de l'enseignement ne peut être effective que si on instaure un régime nouveau et plus réaliste pour l'attribution des bourses, si l'on prévoit l'allocation d'un présalaire au $3^{\mathrm{e}}$ cycle du $1^{\mathrm{er}}$ degré, si l'on considère enfin l'étudiant comme un travailleur, qu'il est en réalité, et qu'on lui alloue un salaire en rapport avec les services qu'il rend et qu'il est appelé à rendre à la collectivité.

Enfin, si l'on veut conserver à l'enseignement sa haute qualité, il est juste de préserver la dignité des maîtres, d'assurer leur prestige social, de favoriser leur perfectionnement professionnel. Il est juste de leur donner une situation matérielle et morale en rapport avec leur valeur technique et humaine et la place éminente qu'ils tiennent dans la vie nationale (1).

(1) Un voeu a été émis par la Commission dans la séance du 14 décembre 1944 demandant la remise en honneur et la revalorisation de la fonction d'enseignement à tous les degrés afin de donner aux membres du personnel, l'indispensable situation morale et l'indépendance qu'exige l'importance de leur rôle social, autant que pour leur assurer un recrutement qualifié surtout dans une période où leur nombre doit être considérablement augmenté.

La réorganisation de la structure générale de l'enseignement et les mesures complémentaires indispensables pour la rendre effective comportent, bien entendu, des conséquences financières. Elles se réfèrent à trois grands chapitres, reconstruction matérielle, aménagement des cadres et financement des mesures de justice sociale.

La réforme de structure exige la révision immédiate de la carte scolaire. L'élargissement des Ecoles maternelles, la prolongation jusqu'à 18 ans de la scolarité obligatoire, l'abaissement de 14 à 11 ans de l'âge de fin de scolarité du premier cycle, le rattachement à l'école des différents 
apprentissages sont des mesures qui conduisent à envisager d'une part un regroupement administratif des établissements existants, d'autre part un plan étendu de constructions et d'équipement scolaires. La reconstruction matérielle de l'école doit accompagner sa réforme administrative et l'extension de son rôle dans la formation économique, civique et humaine de la nation.

Dans les villes et les régions dévastées par la guerre, la reconstruction des bâtiments scolaires doit être envisagée dans le cadre de la réforme de l'enseignement. Il faut considérer par ailleurs que la construction et l'équipement de nouveaux établissements s'intègre dans le plan de reconstruction et d'équipement national. Les investissements nécessaires sont aussi rentables que ceux qui permettront la modernisation de nos services publics et de nos industries. C'est l'école qui assurera la formation d'une main-d'oeuvre qualifiée, de techniciens, de cadres et de chercheurs indispensables au progrès et à la prospérité économique de notre pays, en même temps qu'elle formera des citoyens conscients de leur rôle et de leurs devoirs.

Par ailleurs, le nombre de maîtres à tous les degrés devra être augmenté. La prolongation du temps de scolarité obligatoire, la diminution nécessaire des effectifs par classe, le rôle nouveau assigné à l'école pour la diffusion générale de la culture, la formation technique et civique, ont pour conséquence une nouvelle répartition du personnel actuellement en fonction et la création de nouveaux emplois.

Enfin, le financement des mesures sociales (bourses, présalaire et salaire de l'étudiant) est indispensable pour rendre effective la réforme envisagée.

\title{
CONCLUSION
}

En conclusion, il apparaît extrêmement urgent d'entreprendre les réalisations prévues.

L'application intégrale de la réforme est impossible dans l'immédiat, car elle est liée à des conditions matérielles non encore réalisées. La mise en oeuvre du projet dans sa totalité ne peut se faire que progressivement. Mais un effort doit être fait dès à présent pour l'application d'un certain nombre de mesures, actuellement possibles et orientées dans le sens de la réforme intégrale. Il faut vouloir cet effort sur le plan financier, en nous souvenant que le pourcentage des ressources nationales consacré par la France à l'Education est honteusement faible. En 1946, il n'atteint que 6 à $7 \%$ et nous classe au $26^{\mathrm{e}}$ rang, parmi les nations civilisées, loin derrière l'Angleterre (20\%), les Etats-Unis (21\%), la Russie (25\%). C'est à la fois l'avenir de notre économie et de notre culture qu'il s'agit de préparer et de défendre.

II

\section{STRUCTURE ET ORGANISATION DE L'ENSEIGNEMENT}

\author{
PRINCIPES GENERAUX
}

Le but de l'enseignement est :

$1^{\circ}$ D'assurer aux aptitudes de chacun tout le développement dont elles sont susceptibles. 
$2^{\circ}$ De préparer l'enfant aux tâches professionnelles qui lui sont le plus accessibles et où il pourra le mieux servir la collectivité.

$3^{\circ}$ D'élever le plus possible le niveau culturel de la Nation.

Sauf infirmités assez graves pour lui interdire toute activité culturelle ou professionnelle, tout enfant a droit à l'enseignement sous ces deux formes, qui doivent être données solidairement. Ce sont les services dépendant du ministère de l'Education Nationale qui ont en charge les responsabilités de l'enseignement et de l'éducation à tous ses niveaux et sous tous ses aspects. Ils s'assureront le cas échéant, l'accord ou le concours technique de services dépendant d'autres ministères.

\section{STRUCTURE ET ORGANISATION DE L'ENSEIGNEMENT À SES DIFFERENTS NIVEAUX}

\section{ENSEIGNEMENT DU $1^{\text {er }}$ DEGRE}

Enseignement obligatoire : 6 à 18 ans

L'enseignement se divise en différents cycles où les enfants sont répartis suivant leur âge. Ecole maternelle de 3 à 7 ans.

Les aptitudes des enfants y sont développées librement et sans programme par des méthodes actives.

Remarque. - L'âge actuel d'accueil dans les écoles maternelles est de 2 à 3 ans. Si l'âge d'admission est reporté de 2 à 3 ans selon ce qui a été proposé à la Commission, il sera indispensable pour le bon développement intellectuel des enfants, que les crèches où ils passent souvent toutes leurs journées soient pourvues de jardinières d'enfants.

\section{I - REPARTITION DES CYCLES}

$1^{\text {er }}$ cycle scolaire de 7 à 11 ans.

Remarque. - Dans le cas où il n'y aurait pas de classes maternelles, le cours préparatoire sera confié à une maîtresse initiée aux méthodes actives et l'enfant devra fréquenter l'école dès 6 ans.

$2^{\mathrm{e}}$ cycle scolaire de 11 à 15 ans.

$3^{\mathrm{e}}$ cycle scolaire de 15 à 18 ans.

A l'entrée du $3^{\mathrm{e}}$ cycle (15 à 18 ans) trois embranchements sont possibles :

$1^{\circ}$ Section des études théoriques.

$2^{\circ}$ Section des études professionnelles.

$3^{\circ}$ Section des études pratiques (apprentissage).

Remarque - Une organisation spéciale peut être mise au point dans les milieux ruraux pour que la formation intellectuelle et technique des enfants de 15 à 18 ans ne les empêche pas de travailler effectivement à la terre.

La scolarité obligatoire se trouve ainsi étendue jusqu'à 18 ans. Son terme est aujourd'hui 14 ans. L'allongement n'est pourtant pas aussi considérable qu'il semble. L'enfant ne peut pas actuellement entrer en apprentissage avant 14 ans. Dans le nouveau système il le pourra à 15 . 


\section{II - CARACTERE DE L'ENSEIGNEMENT DONNE AU COURS DES 3 CYCLES D'ETUDES}

\section{$1^{\text {er }}$ cycle de 7 à 11 ans.}

Le même enseignement est commun pour tous les enfants (pour des raisons à la fois psychologiques et pédagogiques) ; mais les méthodes pédagogiques seront en rapport avec les aptitudes variables des enfants. Des écoles spéciales seront organisées d'urgence en nombre suffisant, pour enfants présentant des déficiences mentales et morales et pour infirmes (aveugles, amblyopes, sourds-muets).

\section{$\mathbf{2}^{\mathrm{e}}$ cycle de 11 à 15 ans (cycle d'orientation)}

L'enseignement est en partie commun, en partie spécialisé. L'enseignement commun rassemble tous les enfants, quelle que soit leur spécialisation. (Si des sections distinctes doivent être constituées, ce sera seulement pour tenir compte de la diversité entre les dispositions scolaires des enfants et des différences de méthodes pédagogiques qu'elles peuvent rendre nécessaires). L'enseignement spécialisé comportera un choix d'activités permettant d'éprouver les goûts et les aptitudes des enfants. Ces activités prendront toutes les formes qui peuvent, en rapport avec l'âge, indiquer l'orientation scolaire puis professionnelle qui conviendra ultérieurement à l'enfant. L'âge de la manifestation des aptitudes n'est pas le même pour toutes, certaines paraissent ne pas se révéler avant 13 ou 14 ans. Les options correspondantes ne pourront donc être significatives qu'à cet âge. D'une façon générale il semble que l'assujettissement à des options proprement dites doive intervenir seulement dans les dernières années du $2^{\mathrm{e}}$ cycle, les deux premières années de ce cycle étant plutôt consacrées à une pédagogie active sous la direction de maîtres peu nombreux. Le passage d'une option à une autre devra toujours rester possible grâce à des méthodes rapides de rattrapage. Le rattrapage sera plus facile si, dans chaque option les enfants ne sont pas répartis suivant la classe d'enseignement commun à laquelle ils appartiennent, mais suivant la rapidité de leurs progrès dans l'option. Cette méthode est d'autant plus indiquée que le niveau des aptitudes spéciales dépend beaucoup moins de l'âge que des dispositions individuelles.

Ainsi d'ailleurs pourrait se résoudre le problème des enfants dits "surnormaux". Leur précocité est en général limitée à certaines aptitudes intellectuelles. La maturité d'expérience et de caractère propre aux enfants plus âgés leur faisant habituellement défaut, ils ne seraient pas réunis sans inconvénients pour tout l'enseignement. D'autre part leur rassemblement dans des classes spéciales risquerait d'aboutir à de dangereux forçages intellectuels, sans préjudice de certains risques pour la formation de leur caractère. Au reste, la précocité n'est pas toujours un signe de supériorité définitive.

A mesure qu'elles pourront se préciser d'âge en âge, les options ou les groupements d'options, devront acheminer l'enfant vers une des sections et branches d'enseignement entre lesquelles sera divisé le cycle suivant: section théorique, avec branches littéraires, scientifiques, techniques, section professionnelle et pratique.

Tout enfant doit pouvoir être mis à l'épreuve d'une option quelconque. Il est indispensable que toutes les options figurent dans le même établissement avec l'enseignement commun. Il devra donc y avoir des établissements propres au $2^{\mathrm{e}}$ cycle. Peu importe qu'ils deviennent entièrement distincts des établissements du $3^{\mathrm{e}}$ cycle. Pour des raisons locales certaines options pourront être 
plus développées dans certains établissements : options agricoles à la campagne, options maritimes sur les côtes. Un enfant pourra toujours être muté d'un établissement dans un autre. Les enfants de la campagne devant pouvoir bénéficier de la Réforme comme tous les autres enfants, il faudra distribuer les écoles du $2^{\mathrm{e}}$ cycle sur tout le territoire (écoles cantonales ou intercommunales, avec internats ou ramassage systématique des enfants dans les localités éloignées).

L'importance des remaniements matériels qu'exigera l'organisation correcte de la réforme rendra certains délais nécessaires. Ils doivent être prévus de manière à ne pas être dépassés. C'est un des chapitres de la réforme où devra jouer le système des paliers à échéance déterminée.

\section{$3^{\mathrm{e}}$ cycle de 15 à 18 ans (cycle de détermination).}

A) Section pratique. - Les enfants chez qui les aptitudes manuelles l'emportent sur les aptitudes intellectuelles sont dirigés sur des écoles pratiques d'apprentissage.

L'actuelle obligation d'être présentés devant un centre d'orientation professionnelle reste valable à leur sortie du $2^{\mathrm{e}}$ cycle.

La décision prise à leur égard ne sera pas d'emblée irrévocable. Durant la première année ils devront rester sous le contrôle des orienteurs.

Dans les écoles pratiques d'apprentissage l'enseignement général doit conserver une place importante.

La préparation au métier ne devra pas être étroitement spécialisée ; mais sans oublier que la qualification ouvrière exige l'acquisition de connaissances théoriques et d'habitudes manuelles très précises, on cherchera à préparer les enfants à un secteur d'activité professionnelle aussi ouvert que possible.

Certaines activités industrielles, commerciales ou agricoles n'exigeant pas de qualités professionnelles précises, mais au contraire une aptitude générale à des besognes souvent très variées, il y aura lieu de créer des sections d'apprentissage de caractère polyvalent dans lesquelles l'enseignement sera orienté essentiellement vers la vie pratique.

L'enseignement général doit être autant que possible articulé sur l'apprentissage proprement dit, afin d'avoir une action plus profonde sur l'enfant et d'éviter au métier de devenir une routine sans intérêt et sans autre avenir qu'elle-même.

B) Section professionnelle. - Les enfants susceptibles de fournir les cadres moyens de la production et qui manifestent plus d'aptitudes pour l'exécution que pour les études théoriques seront dirigés sur les écoles professionnelles : écoles commerciales, industrielles, agricoles, artistiques.

Le passage de la section professionnelle dans la section théorique doit rester possible, grâce à des enseignements de rattrapage.

C) Section théorique. - Les enfants dont les aptitudes aux études théoriques auront été reconnues seront dirigés vers les enseignements qui les mèneront au baccalauréat ou aux épreuves qui lui seront substituées.

Ces enseignements seront spécialisés non sans possibilité de contacts entre eux ou de parties communes.

A côté des enseignements de base comme le français, l'histoire, une langue vivante pratique, ils comporteront des groupements à prédominance littéraire, scientifique ou technique. 
Un groupement orienté vers l'étude des langues classiques et la connaissance des civilisations antiques : Humanités classiques.

Un groupement fondé sur l'étude des langues modernes et la connaissance de l'humanité présente : Humanités modernes.

Un groupement tourné vers les sciences d'observation (y compris la physique, la chimie et les mathématiques qui leur sont nécessaires) : Sciences pures.

Un groupement tourné vers les sciences constructives et les techniques y compris physique, chimie et mathématiques : Sciences techniques.

La section théorique achemine vers l'enseignement supérieur mais n'y donne pas directement accès.

\section{ENSEIGNEMENT DU $2^{\mathrm{e}}$ DEGRE}

\section{A - Enseignement propédeutique ou pré-universitaire}

Entre l'enseignement du $1^{\text {er }}$ degré et l'enseignement supérieur s'intercale l'enseignement propédeutique ou pré-universitaire. Il a pour but de rendre à l'enseignement universitaire sa qualité d'enseignement supérieur en donnant aux étudiants la préparation à la fois générale et technique qui leur est nécessaire pour aborder avec fruit l'étude des problèmes qui se posent dans le domaine des sciences ou des lettres.

Il existe déjà en partie sous forme de classes supérieures dans les lycées (première supérieure, mathématiques spéciales) et de P.C.B. dans les facultés des sciences pour la médecine.

Cet enseignement devient obligatoire à l'entrée de tous les enseignements universitaires qui ont pour objet de donner une qualification professionnelle aux étudiants.

Intermédiaire entre l'enseignement des lycées et celui des facultés, il laissera à l'étudiant plus de liberté et d'initiative que le premier, tout en exerçant sur lui un contrôle plus efficace que le second.

Ainsi il ne sera ni annexé aux lycées comme les classes de premières supérieures et de mathématiques spéciales, ni intégré aux facultés comme le P.C.B.

Le personnel enseignant devra répondre à la double exigence de posséder un grand savoir-faire pédagogique et de savoir orienter les esprits dans le sens des études théoriques et scientifiques. Le but de cet enseignement est double : préparation aux études universitaires, première orientation vers la profession. Il devra donc comporter une formation théorique et un premier contact avec les réalités de la profession.

Par exemple les futurs maîtres et professeurs qui feront en commun leurs études propédeutiques dans les écoles normales s'y prépareront chacun suivant sa spécialité aux études supérieures, mais y bénéficieront aussi d'un contact qui ne saurait sans inconvénient être plus tardif avec les élèves des classes annexes. Entre eux pourra se faire la discrimination de ceux qui s'intéressent davantage à l'enfant pour lui-même ou à telle espèce de connaissances.

Pour les futurs médecins une certaine pratique hospitalière prémédicale pourrait s'ajouter aux connaissances générales de biologie, de physique, de chimie qui sont jugées indispensables avant le début des études médicales.

Même remarque pour la profession d'ingénieur, de juriste, etc ... L'enseignement propédeutique donnera à l'étudiant la formation théorique nécessaire en vue des études à entreprendre et une 
première initiation pratique par un contact direct avec l'objet ou les instruments de la profession.

Décisions relatives aux grandes écoles :

$1^{\circ}$ Les grandes écoles deviennent des Instituts d'Université spécialisés rattachés aux Universités.

$2^{\circ}$ Les Instituts spécialisés ne sont ouverts qu'à des candidats pourvus d'une licence qu'ils auront préparée dans une Université.

$3^{\circ}$ Pendant une période transitoire les Instituts spécialisés sont ouverts immédiatement après les années propédeutiques, sous la réserve que les élèves des Instituts poursuivent pendant deux années leurs études de licence dans les Universités ou les Instituts d'Université.

\section{B - Enseignement supérieur}

La Réforme de l'enseignement devra remédier à trois séries d'inconvénients présentés actuellement par l'enseignement supérieur. Il a trois objectifs distincts qu'il confond pour le plus grand préjudice de chacun : formation professionnelle pour les professions essentiellement fondées sur des connaissances intellectuelles ; contribution aux progrès de la science et formation de chercheurs scientifiques ; diffusion de la culture scientifique, littéraire, artistique. Il est actuellement donné par les facultés qui s'ignorent entre elles et par des établissements (grandes écoles et instituts) qui non seulement s'ignorent, mais qui ignorent aussi les facultés. Il est distribué dans des conditions extrêmement inégales par des Universités d'importance très variable et de répartition géographique devenue tout à fait défectueuse.

\section{Regroupement des Enseignements théoriques et techniques dans les Universités.}

Afin d'éviter l'éparpillement des enseignements à travers les Instituts et les Facultés d'une même Université et pour réagir contre leur caractère tantôt trop exclusivement technique et tantôt trop théorique, il convient d'opérer entre eux les coordinations indispensables.

Sans toucher peut-être à l'appareil administratif des Facultés, il faut développer les centres d'études appelés Instituts d'Université qui unifieront les enseignements de même nature ou étroitement complémentaires. Ils pourront ainsi établir des programmes spécialisés d'enseignement et de recherche.

Ils devront jouir d'une autonomie plus grande pour la disposition de leurs ressources, pour leur organisation pédagogique et scientifique.

Mais en même temps chaque enseignement devra rester coordonné avec tous ceux qui contribuent à la même formation professionnelle. Comme il y a actuellement à l'Université de Paris un Institut de Psychologie qui groupe tous les enseignements et tous les laboratoires de psychologie, on pourra envisager par exemple un Institut de Physiologie qui groupera les enseignements de physiologie donnés à la Faculté des Sciences, à la Faculté de Médecine, au Collège de France, au Muséum. Mais chaque enseignement dépendra en même temps soit de la Faculté des Sciences, soit de la Faculté de Médecine, etc. Dans les mêmes conditions pourra se constituer un centre d'études économiques avec participation de la Faculté de Droit, des Sciences et des Lettres.

Il y aura donc coexistence de conseils de Centres d'études et de conseils de Faculté. 
Quant aux écoles ou instituts professionnels ils deviendront des instituts techniques reliés aux Facultés et aux centres d'études. Ils donneront l'enseignement de spécialisation technique tandis que l'enseignement théorique général, jusqu'à présent dispersé entre eux au prix de doubles emplois inutiles et d'insuffisances forcées, sera donné à l'Université ou contrôlé par les examens d'Université.

En principe les étudiants feront deux ans d'études théoriques et pratiques à l'Université correspondant aux années de Licence et recevront ensuite l'éducation technique dans les Instituts spécialisés.

Pour l'établissement des programmes dans ces instituts, le Ministre de l'Education Nationale devra éventuellement obtenir l'accord des Ministères réglant l'activité professionnelle correspondante (Travaux Publics, Agriculture, etc.).

Il sera souvent besoin, dans ces instituts ou écoles, de recruter des maîtres ou moniteurs techniques. Leur choix pourra exiger l'accord du Ministère de l'Education Nationale et de ]'Administration ou des associations professionnelles correspondantes. Leur formation pédagogique sera à la charge de l'Éducation Nationale.

\section{Regroupement régional des Universités.}

Pour donner aux Universités provinciales l'indispensable vigueur que beaucoup d'entre elles n'ont pas, pour leur assurer un personnel enseignant, en laboratoires, en moyens de recherche, les ressources qu'elles ne peuvent avoir, il est nécessaire de les regrouper.

Le regroupement n'implique pas la concentration dans une même ville de tout l'appareil universitaire, ni par suite la suppression dans d'autres villes de toute activité universitaire. Si plusieurs Universités actuelles sont regroupées en une seule, il ne sera pas nécessaire de préparer dans toutes les mêmes licences avec un personnel souvent squelettique ou pour un nombre dérisoire d'étudiants. Réunis, les professeurs de mêmes spécialités ou de spécialités voisines pourront se distribuer entre eux les tâches d'enseignement et de recherche, ce qui sera d'un profit manifeste pour les uns et pour les autres. Dans un des autres sièges de l'Université d'autres tâches seront poursuivies.

Il ne s'agit pas en effet de réduire le nombre de nos établissements ou institutions universitaires déjà insuffisant, ni d'éteindre les foyers de culture là où ils existent, mais bien au contraire d'en développer l'influence et l'action.

En certains cas il pourra paraître nécessaire de concentrer dans la même ville tous les enseignements de licence. Mais les instituts techniques pourront être répartis dans d'autres localités. Et il reste aussi aux Universités deux tâches à remplir : celle de la recherche scientifique et celle de la diffusion culturelle. Ainsi Besançon, dont l'Université est une des plus petites de France, pourrait sans inconvénient pour personne, cesser d'organiser, avec quelles difficultés, tous les enseignements de licence. En revanche, les recherches de chronométrie ou de géologie pourraient y être développées de manière à y attirer les savants français ou étrangers, ou les jeunes gens qui se préparent à ce genre d'études. En outre, il serait possible d'organiser dans toutes les Universités des enseignements proprement culturels qui rayonneraient sur toute la région, et qui seraient un moyen de rassembler les organisations ou les personnalités soucieuses de participer aux grands mouvements intellectuels ou esthétiques de notre époque, soit en y contribuant activement (sociétés de folklore, d'histoire locale, d'exploration géologique, 
botanique, etc), soit seulement en s'efforçant de diffuser dans leur milieu local l'ensemble des connaissances et des manifestations qui constituent notre civilisation toujours en mouvement.

\section{Les trois fonctions de l'enseignement universitaire.}

$1^{\circ}$ ) L'enseignement à objectif professionnel donné dans les Universités puis complété dans les Instituts techniques pourra, dans sa partie théorique, éviter le rétrécissement de l'enseignement général donné aujourd'hui dans les écoles spéciales et, dans sa partie pratique, s'ajuster exactement aux nécessités actuelles de la profession.

Il devra se faire dans toutes les Universités suivant les mêmes programmes. Disparaîtront alors les abus plusieurs fois signalés, quand chaque Université fixe ses programmes de licence. Mais du point de vue technique, il devra constamment être tenu à jour grâce à des rapports réguliers avec la profession et avec la recherche.

$2^{\circ}$ ) La recherche, trop souvent détournée de ses objectifs propres, et bornée dans ses moyens, surtout quand elle s'attache des domaines de la science qui n'ont pas leur place dans les programmes universitaires, recevra une suffisante autonomie pour obéir à ses propres nécessités.

Les futurs chercheurs poursuivront leur formation dans les centres d'études et les instituts d'Universités où ils trouveront les milieux nécessaires à leur éducation scientifique grâce à la collaboration de professeurs et de chercheurs.

$3^{\circ}$ ) L'enseignement purement culturel aura toute liberté pour s'ajuster aux besoins des temps et du milieu. Il ne sera plus limité par le souci de se conformer à ce qui semblait jusqu'à ce jour, représenter la norme universitaire en s'efforçant par exemple de se faire reconnaître le droit à un certificat de licence, ce qui aboutit à encombrer démesurément les programmes scolaires et finalement à desservir l'ordre de connaissances qu'il s'agissait de faire valoir, car c'est à des expériences scolaires qu'elles aussi aboutissent.

L'enseignement culturel pourra accueillir des étudiants qui ne seront pas obligés de passer par l'enseignement pré-universitaire, mais qui pourront avoir d'autres justifications précieuses, par exemple celle d'avoir voyagé, d'avoir participé à des activités économiques, esthétiques ou même sportives, de s'être découvert des curiosités ou des vocations que l'Université peut ne pas avoir eu l'occasion de susciter, mais qu'il peut lui appartenir d'aider à se développer.

L'enseignement culturel ne sera pas nécessairement un enseignement de pure vulgarisation. Il sera seulement celui qui n'a pas à intervenir dans la formation professionnelle donnée par les Universités et qui peut intéresser d'autres esprits que ceux de formation spécifiquement universitaire.

Continuant la vulgarisation et la stimulation des curiosités originales ou rares, le rayonnement de la culture dans les masses et l'appel aux vocations individuelles, l'enseignement culturel devra déborder, dans toute la mesure du possible, l'enceinte des Universités et se mettre en rapport avec tout ce qui peut être foyer de culture ou instrument de recherche : Ecoles Normales de la région, sociétés savantes, groupements d'archéologues, d'architectes, de folkloristes, d'archivistes, de musiciens, etc.

III

FORMATION DES MAITRES 
L'enseignement comprend

$1^{\circ}$ Un enseignement obligatoire jusqu'à 18 ans (ancien primaire et secondaire) ;

$2^{\circ}$ Un enseignement pré-universitaire ;

$3^{\circ}$ Un enseignement supérieur;

$4^{\circ}$ Des enseignements spéciaux ou techniques.

La formation des maîtres va être envisagée successivement pour chacun d'entre eux. Des chevauchements seront d'ailleurs possibles de l'un dans l'autre.

\section{$1^{\circ}$ Enseignement obligatoire}

A l'ancienne distinction entre maîtres du primaire et maîtres du secondaire est substituée la distinction entre maître des matières communes et maître de spécialité.

L'enseignement des matières communes est exclusif dans le premier cycle (6 à 11 ans). Il est partiel de 11 à 15 ans.

L'enseignement des spécialités est partiel de 11 à 15 ans. La spécialisation est complète de 15 à 18 ans.

Futurs maîtres de matières communes et de spécialités suivent les cours des mêmes établissements jusqu'à 18 ans. Ils se présentent. au baccalauréat de leur choix. Un système particulier de bourses pourra être envisagé pour que le recrutement des maîtres puisse rester populaire. Il est d'ailleurs inscrit dans nos principes constitutionnels que nul ne doit être empêché d'atteindre aux postes dont il est digne par défaut d'argent.

Les futurs maîtres de matières communes et de spécialités feront leurs deux années préuniversitaires dans les écoles normales. Ils y recevront une double formation pratique et théorique . D'une part, ils seront mis dans les écoles annexes au contact des écoliers (épreuve qui ne saurait être retardée davantage sans de graves inconvénients pédagogiques). D'autre part, ils seront préparés chacun suivant sa spécialité à l'enseignement théorique des universités. Les écoles normales pourront, dans une certaine mesure, être un cycle d'orientation préuniversitaire. La distinction pourra être faite entre les futurs maîtres qui s'intéressent surtout aux enfants, à leur psychologie, aux problèmes pédagogiques, et ceux que leurs goûts destinent plutôt aux études littéraires et scientifiques.

Les années d'école normale sont suivies pour tous par 2 années de licence à l'université. Après la licence les maîtres seront répartis selon les goûts, les aptitudes, les études faites et les spécialités choisies entre l'école maternelle et les trois cycles du premier degré.

Ils seront stagiaires durant un an et ne seront titularisés qu'après un examen pratique, d'aptitudes pédagogiques. Pour éviter que dans les premières années une prolongation des études n'entraîne une pénurie de maîtres, là comme ailleurs des paliers pourront être envisagés dans l'exécution de la Réforme.

Les licenciés pourront préparer une agrégation réformée selon les besoins nouveaux (qui associera à de hautes connaissances scientifiques une culture pédagogique), soit après la licence, soit après quelques années d'exercice. Durant l'année de préparation, ils seront déchargés d'enseignement. La préparation de chaque agrégation sera organisée dans les Universités par un groupement approprié des enseignements. Les agrégés enseigneront d'abord dans les $2^{\mathrm{e}}$ et $3^{\mathrm{e}}$ cycles avant d'enseigner éventuellement dans le cycle pré-universitaire.

\section{$2^{\circ}$ Enseignement pré-universitaire. Ecoles normales supérieures}


Pour enseigner dans le cycle pré-universitaire il faudra être agrégé.

Les écoles normales supérieures (E.N.S.) sont des établissements de haute culture. On n'y entrera qu'après les deux années universitaires et la licence. Elles comporteront une section d'élèves recrutés par concours et une section recrutée parmi les maîtres déjà en exercice. Dans les E.N.S. littéraires et scientifiques (type rue d'Ulm et Sèvres), les élèves se familiariseront avec les méthodes de recherche qui leur permettront ultérieurement de préparer des travaux en vue de l'Enseignement supérieur.

Les E.N.S. pédagogiques (type Saint-Cloud et Fontenay, car les besoins obligeront à en créer d'autres dans les grandes villes), formeront leurs élèves à la recherche pédagogique et les destineront naturellement après qu'ils auront enseigné, aux postes de directeurs, d'inspecteurs, de professeurs de pédagogie dans les écoles normales.

L'E.N.S. d'enseignement technique formera elle aussi des inspecteurs, des directeurs de centres. Elle restera provisoirement un institut technique de l'Université comme les grandes écoles professionnelles rattachées à l'Université : Mines, Travaux Publics, Ponts et Chaussées, etc ... L'E.N.S. d'éducation physique jouera un rôle analogue dans le domaine des activités physiques éducatives.

Il ne paraît pas indiqué de terminer les années d'E.N.S. par un concours. Elles doivent plutôt donner lieu à des mémoires ou à des rapports et à la collation d'un grade.

La durée des études pourra y être variable suivant la spécialité.

\section{$3^{\circ}$ Enseignement supérieur}

Actuellement, le mode de formation et de recrutement n'est pas le même pour les facultés des Lettres, des Sciences, de Droit, de Médecine et de Pharmacie.

Les facultés de Lettres et de Sciences recrutent leurs maîtres parmi les docteurs ès Lettres ou ès Sciences qui ont été inscrits sur la liste d'aptitude à l'enseignement supérieur; les facultés de Médecine, par un concours sur le plan local bien qu'avec un jury unique pour toute la France ; les facultés de Droit par un concours national.

Il n'y a pas actuellement de formation prévue pour les maîtres des Lettres et des Sciences. Ils sont très diversement aidés et guidés dans la réalisation de la thèse qui doit leur ouvrir les portes de l'enseignement supérieur : les uns ont des postes dans des laboratoires de recherche et peuvent y trouver les appuis nécessaires, les autres sont livrés à eux-mêmes. Les écoles normales supérieures pourraient aider à combler cette lacune. Il n'y a pas lieu de prévoir un concours d'entrée dans l'enseignement supérieur des Lettres et des Sciences.

Dans les facultés de Médecine, les candidats au concours d'agrégation ont été le plus souvent chefs de clinique et se sont ainsi préparés aux fonctions d'enseignement. Le concours comporte une épreuve sur titre, une épreuve clinique et des leçons. Son vice est d'opérer sur le plan local, et par suite de limiter le choix, d'éliminer souvent les meilleurs et de rendre possible le favoritisme régional ou familial.

De hautes autorités médicales demandent que le concours ait lieu désormais sur le plan national. Les candidats reçus à l'agrégation seraient nommés à l'un des postes vacants de leur spécialité, dans une faculté quelconque. Ils auraient droit dans la ville où ils seraient envoyés à un service d'hôpital. Ils pourraient se consacrer exclusivement à ce service et à leur enseignement et recevraient un traitement en conséquence. Ils pourraient être autorisés à faire de la clientèle, mais ne recevraient dans ce cas qu'un traitement partiel. Ils pourraient être dans la suite appelés à exercer dans une autre université suivant le même mode de désignation que pour les autres facultés. 
Pour les candidats à l'agrégation de Droit, la préparation est assurée par les professeurs de faculté, mais en dehors de leur service obligatoire qui est entièrement consacré au cours de spécialité. Ce cours doit être fait complètement chaque année. Il pourrait être en bien des cas, suppléé ou complété par des manuels. Le temps devenu ainsi disponible serait employé à un enseignement vraiment supérieur et à la préparation officielle de l'agrégation.

\section{$4^{\circ}$ Enseignements spéciaux}

Les enseignements spéciaux sont ceux pour lesquels l'Université doit recruter des maîtres qui aient l'expérience de l'industrie, de l'agriculture, des affaires, de l'administration, bref, de la profession sous toutes ses formes et à tous ses niveaux. A l'échelon de l'enseignement supérieur il s'agit des instituts techniques d'Université, aux échelons plus bas des écoles professionnelles, des centres de formation agricole, des écoles pratiques d'apprentissage.

Ces maîtres pourront, s'il est besoin, recevoir une formation pédagogique, par exemple en participant à des stages dans des établissements appropriés.

\section{IV \\ ORGANES DE CONTROLE ET DE PERFECTIONNEMENT}

L'Université réformée doit pouvoir contrôler et perfectionner ses activités. Le contrôle doit être pédagogique sur les maîtres et psychologique sur les élèves.

$1^{\circ}$ Le contrôle pédagogique existe déjà sous la forme de l'inspection, mais elle doit être réorganisée pour répondre pleinement à son but. Trop souvent, l'inspecteur est absorbé par des fonctions purement administratives aux dépens des tâches pédagogiques. Tout ce qui n'est pas en connexion étroite avec ses responsabilités pédagogiques devra être confié à des services purement administratifs. D'autre part, tout inspecteur devra pouvoir être aidé par un secrétariat dont l'importance sera en rapport avec le volume de ses obligations administratives.

Aujourd'hui, l'inspecteur, faute de temps sans doute, est bien plus le juge que le guide des maîtres qu'il inspecte. Il manque ainsi à l'essentiel de sa fonction. Il serait plus utile d'améliorer l'enseignement donné aux élèves que de classer les maîtres entre eux. Il faut que l'inspecteur devienne le conseiller permanent de ses administrés, qu'il répande parmi eux la connaissance des progrès que peut réaliser la pédagogie et qu'il soit capable de leur en montrer les applications. Pour être à la hauteur de cette mission, l'inspecteur ne devra pas s'en tenir à son expérience personnelle ni à une doctrine une fois pour toutes élaborée. En même temps qu'il exercera ses fonctions d'inspecteur, il restera en collaboration constante avec les centres de recherches pédagogiques.

Pour répondre à toutes ces conditions, il convient que l'inspecteur ait, de l'enseignement qu'il aura le devoir de contrôler, une expérience personnelle, c'est-à-dire qu'il l'ait lui-même exercé pendant une période d'au moins dix ans. Il faudra donc dans certains cas des inspecteurs spécialisés. Ainsi évitera-t-on l'hérésie, par exemple, d'assimiler l'enseignement des anormaux et celui des écoles maternelles, en donnant au premier les mêmes inspecteurs qu'au second. Le postulant à un poste d'inspecteur devra faire un stage suffisamment prolongé dans un établissement de hautes études pédagogiques, c'est-à-dire à l'école normale supérieure du type de 
Saint-Cloud ou à l'école normale supérieure du type de Fontenay, devenues les centres les plus élevés de la formation pédagogique et des recherches sur l'éducation.

Suivant leur rang, les inspecteurs devront collaborer aux activités pédagogiques de ces écoles ou des centres de recherche et d'expérimentation qui seront créés auprès des universités ou dans les écoles normales.

$2^{\circ}$ Le contrôle psychologique s'adresse aux élèves. Il n'existe encore à titre d'expérience et officieusement que dans quelques groupes scolaires de la région parisienne ou de la province. Il répond à la nécessité de connaître l'enfant dans ses particularités individuelles aussi bien que dans son évolution psychologique. Les fonctions d'enseignement sont trop absorbantes pour laisser aux maîtres le loisir d'étudier et d'appliquer les méthodes d'investigation qui permettent de déterminer éventuellement pour chaque enfant les causes intellectuelles, caractérielles ou sociales de son comportement scolaire. Ils doivent pouvoir soumettre le cas à un spécialiste des méthodes psychologiques. Ces examens psycho-techniques devront contribuer à l'orientation scolaire des enfants.

D'autre part, il faut pouvoir apprécier les conséquences psychologiques des méthodes éducatives. Le bon rendement scolaire n'est pas toujours un critère suffisant. Certains procédés pédagogiques peuvent être très efficaces, mais au prix d'une plus grande fatigue pour l'enfant ou au détriment d'autres aptitudes utiles, telles que la spontanéité, l'initiative, etc. Des épreuves psychologiques bien choisies peuvent seules permettre d'établir ce bilan.

Enfin, les programmes eux-mêmes doivent être pour chaque classe adaptés aux aptitudes propres à chaque âge, et sans cet ajustement il n'y a pas de précision possible sans emploi de critère psychologique.

Il est donc indispensable de prévoir, comme l'ont fait les réorganisateurs de renseignement dans d'autres pays, la création d'un corps de psychologues scolaires. Ils devront avoir une justification pédagogique suffisante (diplômes universitaires, et, s'il se peut, pratique scolaire). Ils devront en outre avoir suivi un enseignement théorique et pratique de psychologie que sanctionnera un diplôme reconnu par l'Etat et délivré par l'Université tel que le diplôme de psychologie pédagogique actuellement délivré par l'Institut de Psychologie de l'université de Paris.

Ils exerceront leurs fonctions, comme le personnel enseignant, sous la responsabilité des autorités universitaires. Ils seront en outre soumis au contrôle technique des laboratoires ou centres d'études psychologiques existant ou à créer auprès des universités.

Leur statut et les règles de leur avancement devront tenir compte de leur double qualification pédagogique et psychologique.

A ce contrôle psychologique devra s'ajouter un contrôle physiologique qui rentre dans les attributions des médecins scolaires. Ils ne se borneront plus au dépistage rapide de certaines maladies, mais ils devront suivre la croissance de chaque enfant et se tenir en rapport avec les maîtres et les psychologues pour noter les concomitances qui pourront s'observer dans son comportement scolaire, psychique et biologique. 
L'organisation de l'enseignement pose trois problèmes solidaires mais distincts.

\section{PROGRAMMES}

Les programmes doivent indiquer ce qui sera enseigné par cycle et par année, mais de façon large et souple, c'est-à-dire que la distribution dans le temps de leurs matières ne doit pas être trop stricte, afin de ne pas contrarier, par un compartimentage excessif, l'élan de curiosité qu'il faut chercher à susciter chez les enfants, car c'est là un des buts essentiels de l'éducation si elle veut être pleinement profitable.

Ils ne fixeront pas non plus de façon irrévocable le détail des matières à enseigner, car il peut être avantageux de ne pas disperser à l'excès l'intérêt des élèves et il est parfois préférable, pour la meilleure compréhension ou la meilleure utilisation d'une discipline, d'insister davantage sur une question que de se borner à les résumer toutes. Pour la bonne formation de l'esprit, il n'est pas besoin de connaissances encyclopédiques, mais de connaissances en profondeur.

Les programmes comportent un programme commun dans le premier cycle du premier degré (cycle élémentaire) et partiellement commun auquel s'ajoute un programme partiellement spécialisé dans le second cycle (cycle d'orientation). Dans le troisième cycle (cycle de détermination), l'orientation est à peu près fixée. Les seules matières communes seront l'étude du français, d'une langue vivante pratique, de l'histoire, du dessin, de la musique et du chant. Le contenu de l'enseignement commun sera, au cours du premier cycle (6-11 ans), ce qui répond aux instruments indispensables de la connaissance (lecture, écriture, calcul), ce qui enrichit les moyens d'expression (dessin, langage) et toutes les activités qui peuvent développer les aptitudes d'observation. Cet âge étant particulièrement propice à l'étude des langues, il conviendra dans toute la mesure du possible, d'apprendre aux enfants une langue étrangère d'usage courant, par la méthode directe.

Dans le deuxième cycle, l'enseignement commun comportera l'étude de la langue française et d'une langue étrangère, une formation intuitive aux mathématiques, l'observation du milieu, et des notions d'histoire et de géographie susceptibles de développer chez l'enfant le sens des différences d'espace et de temps.

C'est avec le deuxième cycle que commencent les options. Pendant les deux premières années, elles consisteront en activités d'essai qui serviront aux maîtres à mieux connaître les enfants. Dans les années suivantes, le choix de ceux-ci sera plus libre. Les groupements optionnels se répartiront en scientifiques, littéraires, techniques, artistiques. Suivant le temps qui leur sera consacré, les options seront distinguées en options principales et options secondaires. C'est parmi les options que figurera la langue classique ou moderne, dont l'étude sera faite non plus par la méthode directe, mais par les méthodes grammaticales, philologiques, littéraires, historiques, qui en feront un instrument de culture. Parmi les options figureront également les mathématiques rigoureuses, les techniques d'observation et en général tout ce qui peut déceler et développer des aptitudes spécialisées.

Durant le troisième cycle, les élèves pourront choisir des options complémentaires, mais suivant leurs spécialisations de base, certains groupements d'options seront obligatoires. Un enseignement d'hygiène sera donné pendant la dernière année du troisième cycle.

HORAIRES 
L'horaire doit fixer le nombre total des heures consacrées à l'enseignement et la distribution de ces heures entre les différents enseignements.

Pour fixer le temps consacré par l'enfant à l'école, il faut tenir compte de ses possibilités physiologiques et de ses besoins psychologiques. Il ne devra pas excéder, entre 7 et 9 ans, 2 heures par jour et 10 heures par semaine ;

De 9 à 11 ans : 3 heures par jour et 15 heures par semaine ;

De 11 à 13 ans : 20 heures par semaine.

De 13 à 15 ans : 25 heures par semaine.

Dans cet horaire seront incluses les séances de "travail dirigé'. Ce travail sera véritablement dirigé de 11 à 15 ans, il sera seulement contrôlé durant le troisième cycle et complètement libre dans les dernières années. En dehors de la classe, l'enfant devra pouvoir se livrer à toutes les activités de son choix : jeux, lectures, etc.

Quant à l'emploi du temps à l'école, il a évidemment une triple utilité : équitable répartition des journées scolaires entre toutes les disciplines, exacte articulation des enseignements, ce qui est particulièrement nécessaire quand ils sont distribués entre des maîtres différents, contrôle facile de leur correcte exécution.

Néanmoins, il doit, lui aussi, être assez souple pour ne pas morceler ni lasser l'attention des enfants. Ce résultat sera surtout obtenu par l'entente entre les maîtres, qui devront se concerter dans les conseils de classe, dont les réunions périodiques seront fréquentes. L'horaire prescrit devra être plus indicatif que rigide.

\section{METHODES}

Les programmes étant fixés pour chaque âge et selon chaque orientation des enfants, il appartiendra aux méthodes d'en ajuster l'exécution aux capacités de chacun. Les méthodes à utiliser sont les méthodes actives, c'est-à-dire celles qui s'efforcent d'en appeler pour chaque connaissance ou discipline aux initiatives des enfants eux-mêmes. Elles alterneront le travail individuel et le travail par équipes, l'un et l'autre étant susceptibles de mettre en jeu les différentes aptitudes de l'enfant, tantôt en lui faisant affronter avec ses ressources propres les difficultés de l'étude, et tantôt en lui faisant choisir un rôle particulier et une responsabilité personnelle dans l'oeuvre collective. Ainsi se révéleront ses capacités intellectuelles et sociales, et la place laissée à sa spontanéité fera de l'enseignement reçu un enseignement sur mesure.

\section{SANCTION DES ETUDES}

Aucun examen ou concours ne doit avoir lieu avant la fin des années de scolarité obligatoire. Le groupement des élèves dans le $2^{\mathrm{e}}$ cycle du $1^{\mathrm{er}}$ degré, leur répartition dans les différentes sections du $3^{\mathrm{e}}$ cycle dépendront de leurs activités antérieures et de l'avis du conseil des maîtres (orientation) à qui incombera la décision.

Les examens à tous les degrés devront être conçus sur un type nouveau qui dissocie les épreuves de connaissances et l'appréciation des aptitudes. Les examens de fin d'études doivent être un contrôle portant sur l'ensemble des études et ne doivent pas permettre la préparation spéciale d'un programme limité. Par des sondages multipliés on s'efforcera d'explorer l'ensemble des acquisitions et des aptitudes en éliminant le plus possible le hasard. 
Ces examens de $\mathrm{f}$ in d 'études porteront sur les enseignements généraux dans les enseignements spéciaux à chaque section du $3^{\mathrm{e}}$ cycle.

Ils donneront lieu à l'attribution, dans la section pratique du certificat d'aptitude professionnel, dans la section professionnelle, du brevet d'enseignement professionnel ; dans les sections théoriques, du baccalauréat classique, technique, moderne, etc.)

Le baccalauréat permettra l'entrée dans l'enseignement pré-universitaire. Un stage probatoire de courte durée servira à diriger les étudiants vers les enseignements en rapport avec leurs aptitudes et, si besoin est, à éliminer les inaptes.

Des concours donneront accès aux instituts et aux grandes écoles. Ils devront être autant des épreuves de caractère et d'aptitude que des épreuves de connaissances. Ils seront institués au moment de l'entrée à l'Université, mais ne dispenseront pas les élèves reçus de faire à l'Université leurs années de licence. Le recrutement se complétera par un second concours ouvert aux candidats achevant la licence. Aucun étudiant ne pourra se présenter plus de deux fois : une fois à la fin des années pré-universitaires, une fois à la fin des années de licence. Au delà de la licence, l'agrégation sera organisée conformément aux dispositions prévues dans le chapitre : Formation des Maîtres.

Des doctorats d'Université, de philosophie, histoire, physique, géologie, etc, de même caractère que le Doctorat en médecine ou en Droit, seront attribués après deux ou trois années d'études supérieures sur la présentation d'une thèse.

Les Doctorats ès Lettres ou ès Sciences conservent leur ancienne valeur de haute qualification scientifique.

\section{APPENDICE}

\section{SECTIONS DE RATTRAPAGE}

Mais à côté de la diversité qualificative qui peut exister entre les esprits, il peut y avoir une différence dans le niveau d'intelligence globale. Il ne doit plus en résulter comme aujourd'hui qu'un enfant dont le développement ou la puissance intellectuelle sont médiocres, "redouble" ses classes et devienne le condisciple d'enfants qui ont deux ou trois ans de moins que lui. Car, à bien des égards, il n'est plus leur semblable. Son expérience de la vie courante est plus développée, ses intérêts deviennent différents et on le retient devant les mêmes difficultés, dans la forme exactement où elles l'ont déjà rebuté. Au lieu d'utiliser et de stimuler ses actuelles dispositions psychiques, qui pourraient l'aider à surmonter l'obstacle, on prétend mettre en oeuvre celles qui risquent d'appartenir à un âge déjà révolu et qui peuvent être sur leur déclin. Il n'y a aucune raison pour que le programme, s'il est bien adapté à l'âge des enfants, ne reste pas le même pour tous ceux du même âge, mais à condition d'être allégé, si c'est nécessaire, et surtout d'être enseigné par des méthodes qui sachent exploiter à fond les dispositions et les goûts les plus développés de chacun. C'est là une tâche pédagogique considérable mais urgente. Elle exige évidemment la collaboration des pédagogues et du psychologue scolaires.

Il pourra donc être indiqué de constituer des sections particulières pour les enfants qui se révéleraient incapables de suivre le cours normal des études, sans y subir un retard dans leur progression de classe en classe. Un sondage récent fait dans les écoles primaires de la région parisienne a montré que $22 \%$ des enfants sont plus âgés de 2, 3 ou même 4 ans qu'ils ne 
devraient l'être dans les classes qu'ils fréquentent. Cette situation doit cesser. Chaque fois, d'ailleurs, que la chose redeviendra possible, l'enfant retournera de la section de rattrapage dans une classe normale.

\section{SECTIONS DE PERFECTIONNEMENT}

\section{$1^{\circ}$ Pour les déficients intellectuels}

Un cas extrême est celui dés enfants intellectuellement déficients, c'est-à-dire qui sont véritablement et définitivement incapables d'assimiler le programme commun, mais pour lesquels il y a des possibilités d'adaptation sociale et professionnelle et qui doivent, par conséquent, être mis en état de se suffire un jour à eux-mêmes.

Il faut pour eux un enseignement spécial, comme l'avait prévu la loi de 1909 dont l'application n'était malheureusement que facultative. Le nombre de ces enfants est actuellement, d'après le sondage dont il a été parlé, de $8 \%$.

Ces enfants ont droit à l'instruction. Ils ne doivent pas non plus rester une charge sans compensation dans une société qui a tant besoin de main-d'oeuvre. Il s'agit de leur donner les instruments intellectuels indispensables et l'habitude des travaux qui leur seront accessibles. Il serait chimérique de subordonner cette formation scolaire et professionnelle à l'emploi des méthodes fondées uniquement sur le développement des connaissances par la culture intellectuelle. En bien des cas, il faudra se résigner à un dressage par l'habitude et par la mémoire. Les seules chances de culture, dans le cas d'une intelligence déficiente, c'est l'accès possible à la vie de tous, à l'expérience commune, c'est-à-dire de ne pas être condamné à l'isolement par une impéritie restée totale.

Cet enseignement spécial exige beaucoup de discernement, car il doit savoir doser pour chaque enfant le dressage et l'éveil intellectuel, qui demeure évidemment le but essentiel. Mais cet éveil intellectuel doit avoir d'autres stimulants que pour les enfants intelligents. Il doit faire participer l'enfant à des situations concrètes sur lesquelles pourront s'ajuster son savoir-faire et consécutivement sa compréhension.

Il faut donc développer son savoir-faire. Il sera indispensable d'introduire dans la section de perfectionnement, avec les exercices de lecture, d'écriture, de calcul, de dessin qui y sont déjà pratiqués, les travaux manuels, qui le sont insuffisamment. La collaboration entre maîtres de l'enseignement commun et maîtres de l'enseignement technique devient ici aussi nécessaire. L'enfant déficient doit bénéficier d'un préapprentissage commencé de bonne heure et poursuivi jusqu'à 18 ans. Souvent même sera-t-il nécessaire qu'il soit admis à travailler encore deux ou trois ans dans des ateliers spéciaux. Son utilisation ultérieure devra être réglée en accord avec des représentants de syndicats patronaux et ouvriers.

L'enseignement spécial est déjà organisé, et il a derrière lui une expérience précieuse. Mais le nombre des classes et des établissements où il est donné, reste notoirement insuffisant. Le nombre de maîtres qualifiés est dérisoire en regard des besoins. Il faut donc en former un très grand nombre, et de toute urgence.

La méthode la plus rapide, la moins onéreuse, la plus efficace, c'est d'organiser des stages de six mois, qui pourraient être, pour commencer, ramenés à trois mois, ce qui représente un minimum à peine suffisant . Il serait d 'un luxe véritablement inopportun de créer une école normale nationale pour maîtres d'arriérés. Son rendement resterait forcément d'une ridicule insuffisance. Mais il faudra avoir des classes ou des internats de perfectionnement avant même d'avoir le nombre de maîtres nécessaires. On le pourra en généralisant une tentative récente qui a donné de 
bons résultats. Des maîtres particulièrement expérimentés dans l'éducation des déficients intellectuels reçoivent une mission d'instructeurs. Ils vont, pour des périodes variables de temps, montrer aux maitres sans formation préalable comment ils peuvent organiser leur classe et instruire leurs élèves. Parmi ces instructeurs, il serait possible de sélectionner des inspecteurs compétents.

L'extension rapide de l'enseignement spécial est le seul moyen :

$1^{\circ}$ d'arracher à une oisiveté nocive pour eux-mêmes et pour la société des enfants que leurs insuccès scolaires obligent à éliminer de l'école.

$2^{\circ}$ de soulager les classes normales du poids qui résulte pour elles d'un nombre parfois important d'élèves incapables d'y faire aucun progrès.

\section{$2^{\circ}$ Pour les déficients sensoriels}

C'est depuis de longues années déjà que le cas des déficients sensoriels s'est imposé à l'attention publique. Il existe des instituts pour les sourds-muets, les aveugles et quelques classes spéciales pour les amblyopes. Cependant, les efforts sont restés très dispersés et l'éducation donnée de niveau très variable et de principes souvent contestables.

L'Education nationale ne peut pas rester indifférente aux problèmes que pose la réadaptation des déficients sensoriels au milieu professionnel, social, culturel où ils doivent pouvoir trouver les meilleures conditions de vie compatibles avec leur infirmité. C'est à l'Education nationale qu'incombe la mission d'éducation pour tous les enfants. C'est à elle seule qu'il est possible d'instituer des expériences et des comparaisons pédagogiques suffisamment étendues pour éviter les erreurs où peut entraîner la vision trop exclusive de certaines insuffisances pathologiques. C'est elle seule qui peut disposer de tous les éducateurs dont la collaboration est nécessaire pour un réajustement aussi étendu que possible à la vie commune de tous ceux qui s'en trouvent exclus par la surdité ou la cécité.

L'Education nationale prendra le contrôle et la charge de tous les établissements existant et en coordonnera l'enseignement selon des buts et avec des méthodes bien étudiés.

\section{SECTION DE READAPTATION}

Une autre catégorie est celle des enfants que leur conduite irrégulière ou leurs délits mettent en conflit avec la société. Leur diversité est grande. Pour déterminer le cas de chacun et le régime qui lui conviendra, il faut prévoir des centres d'observation et de triage où leur séjour ne devra pas dépasser les quelques semaines strictement indispensables aux pédagogues, psychologues, médecins, magistrats chargés de leur examen, car rien ne leur est plus mauvais qu'une trop longue période d'attente et d'indécision.

S'il y en a qui sont jugés comme d'intelligence trop déficiente ou de perversité trop essentielle pour être jamais récupérables, ils seront placés dans des sections appropriées d'asiles. Pour ceux dont la rééducation doit être tentée, le but doit être de les remettre aussi vite que possible soit dans des établissements de déficients simples, s'ils sont d'intelligence déficiente, ce qui est souvent le cas, soit dans des établissements d'enfants normaux, s'ils sont d'intelligence normale. Rien ne saurait mieux les stimuler que ce reclassement qu'il faut leur faire mériter. Rien au contraire n'est plus pernicieux que de laisser se cristalliser dans des établissements spéciaux, si bien organisés soient-ils, leurs communes rancoeurs contre une société dont ils oublient rarement qu'ils ont été les exclus. 
Cependant, il faut des établissements de passage où ils seront mis en état de tenter leur chance, toutes leurs chances selon leurs aptitudes et leur mérite. L'enseignement devra y être varié et comporter en particulier une part très large d'enseignement manuel. L'enfant irrégulier est souvent instable ou a pris des habitudes d'instabilité. Les disciplines abstraites le rebutent, il se plaît au contraire aux réalisations concrètes de son activité. Il est très important cependant qu'il ne se sente pas nécessairement voué aux centres d'apprentissage ou aux écoles professionnelles. Il faut lui retirer tout prétexte de se considérer comme moins libre de son destin que tout autre enfant. A lui seul de fournir la preuve de ses capacités et de son mérite.

Ces établissements devront être en étroite et constante relation avec les autres établissements d'enseignement. L'échange des élèves pourra se faire éventuellement dans les deux sens. Les maîtres devront eux-mêmes pouvoir passer des uns dans les autres s'ils en éprouvent le désir ou le besoin. Seule l'Education nationale est capable d'assurer la coordination de leurs enseignements, le recrutement et la formation des maîtres qualifiés, c'est donc à elle d'assurer la charge de ces établissements.

\section{SECTIONS AUXILIAIRES}

Pour des raisons d'ordre social ou professionnel, il y a des catégories d'enfants à qui la fréquentation de l'école est difficile ou impossible. Par exemple, ceux dont les parents mènent une vie nomade : forains, bateliers, ou ceux que leur état oblige à une vie recluse dans des locaux ou des stations isolés. Ils ont pourtant droit, eux aussi, à tous les bénéfices utilitaires et culturels de l'instruction.

Pour les forains véritablement nomades et dont les déplacements sont habituellement massifs d'une localité vers une autre, la meilleure solution serait de généraliser la création d'écoles qui les accompagnent.

Pour les bateliers, l'organisation d'écoles dans les ports où ils séjournent parfois assez longtemps ne paraît pas suffisante. Car pendant la période de voyage l'enfant risque d'oublier tout l'enseignement reçu. Créer dans ces ports des internats où il pourrait être laissé par ses parents pendant leurs voyages parait être la solution la plus facile à leur faire accepter. Sans doute la création d'internats spéciaux pour enfants de bateliers est en opposition avec le principe d'unification sociale et nationale que l'Education doit poursuivre. Mais les familles de bateliers forment comme des cellules qu'il est difficile de dissocier; les parents se séparent avec peine des enfants et les enfants des parents. Il faut leur rendre cette séparation indispensable aussi acceptable que possible par un internat à régime aussi souple que possible.

Pour les enfants d'hôpitaux, de sana, de préventorium, la tache essentielle est de préparer des maîtres qui sauront prendre toutes les initiatives nécessaires sous la direction des médecins pour que chaque enfant reçoive le maximum d'instruction, avec le minimum de fatigue : horaires, méthodes, programmes devront être étroitement subordonnés aux exigences physiologiques de chacun et tendre davantage à tenir en éveil les aptitudes intellectuelles qu'à distribuer systématiquement un bagage déterminé de connaissances.

Des types de programmes et horaires ont été étudiés par la 2e S/C. sous la présidence de M. Piéron ; ces pièces figurent dans les archives où elles pourront être consultées. 


\section{EDUCATION MORALE ET CIVIQUE \\ FORMATION DE L'HOMME ET DU CITOYEN}

L'Ecole publique comme l'Etat lui-même, aux termes de la Constitution, est laïque, c'est-à-dire que, ouverte à tous les enfants, elle ne peut et ne doit donner aucun enseignement doctrinal, politique ou confessionnel.

La laïcité de l'école n'implique pas qu'elle n'exerce aucune action éducative. S'il ne lui appartient pas d'assurer le recrutement de tel ou tel groupement religieux ou politique, elle doit à la Nation de préparer l'enfant à prendre conscience du rôle qui sera le sien dans la vie sociale et de sa responsabilité de citoyen.

L'éducation morale et civique que l'école doit donner ne saurait se borner à l'étude d'un programme en un temps fixé par l'horaire. On ne peut en effet dissocier l'éducation de l'intelligence de celle du caractère. C'est la vie scolaire tout entière qui offre les moyens d'élever l'enfant. Le contenu de l'enseignement, plus encore ses méthodes et la discipline scolaire, sont les moyens permanents et normaux de donner à l'enfant le goût de la vérité, l'objectivité du jugement, l'esprit de libre examen et le sens critique qui feront de lui un homme libre du choix de ses opinions et de ses actes, de lui faire acquérir le sens de la vie sociale, des avantages et des charges qu'elle implique, et la conscience de ses responsabilités. "L'école, dit Paul Langevin, est une véritable entreprise de culture dont l'individu ne profite pleinement que s'il est entraîné et soutenu par le milieu scolaire. L'école fait faire à l'enfant l'apprentissage de la vie sociale et, singulièrement, de la vie démocratique...Ainsi se dégage la notion du groupe scolaire à structure démocratique auquel l'enfant participe comme futur citoyen et où peuvent se former en lui, non par les cours et les discours, mais par la vie et l'expérience, les vertus civiques fondamentales : sens de la responsabilité, discipline consentie, sacrifice à l'intérêt général, activités concertées et où on utilisera les diverses expériences de "self-government" dans la vie scolaire".

Les mêmes méthodes, basées sur l'activité et l'expérience personnelles qui seront employées pour assurer à l'enfant, dans les meilleures conditions, le maximum de développement physique et intellectuel seront donc utilisées pour sa formation civique et morale. Cette unité des méthodes et l'emploi de moyens en rapport avec le développement psychologique progressif de l'enfant sont les garants de l'efficacité de l'éducation à l'école.

A l'école maternelle, et au début du premier cycle du premier degré, le but principal de l'éducation sera la formation des habitudes : habitudes physiques, habitudes de sociabilité qui orienteront l'enfant vers la conscience de l'existence d'autrui et le respect de ses droits. A cet âge, l'affectivité commande directement l'action : c'est sur elle que l'éducation s'appuiera. Toute l'organisation de la vie scolaire devra favoriser au maximum l'affirmation de la personnalité et du caractère, l'action volontaire, l'initiative et le goût de l'effort. Les méthodes d'individualisation de l'enseignement, d'auto-discipline, le jeu des responsabilités individuelles dans l'organisation matérielle du milieu scolaire seront propices à cette formation. Les règles imposées seront réduites au minimum pratiquement indispensable. Progressivement, on laissera à chacun une part d'activité libre et de responsabilité personnelle de plus en plus large. En même temps, les travaux en équipe, la coopération organisée enseigneront par l'action la soumission volontaire à une règle acceptée, l'intégration de l'activité individuelle à celle d'un groupe organisé. La réflexion abstraite n'étant pas de cet âge, l'enseignement moral théorique sera exclu : la réflexion et le jugement moral s'exerceront sur les faits précis de la vie individuelle et collective. 
Vers la fin du premier cycle, et surtout aux $2^{\mathrm{e}}$ et $3^{\mathrm{e}}$ cycles, le développement psychologique de l'enfant permettra de multiplier les moyens d'action. Le principe en demeurera l'activité propre de l'élève, par l'expérience et la réflexion personnelles. Mais celle-ci pourra s'attacher à des problèmes de plus en plus complexes et débordant le cadre de la vie scolaire.

A ce niveau, le développement intellectuel jouera un rôle important. Toute l'éducation intellectuelle, dans toutes les disciplines, servira la formation morale et civique par la culture de l'esprit critique et le libre examen. Les disciplines scientifiques joueront un rôle éminent si elles donnent le goût de la précision et de la clarté, l'habitude d'éviter la précipitation du jugement, de pratiquer l'objectivité et l'impartialité.

L'élargissement de l'horizon et la préparation à la vie dans la cité se feront par l'initiation progressive (le plus souvent possible par contact direct) à la réalité sociale. C'est moins par les livres et les cours que par les visites, les enquêtes, les recherches personnelles que les adolescents seront conduits à l'analyse critique de la structure sociale, administrative et politique. Une place importante sera faite à la connaissance des professions, de leur rôle, de leur évolution, des conditions où elles s'exercent. L'exacte appréciation du rôle fondamental des travailleurs, de la solidarité des diverses activités humaines orientera les adolescents vers la compréhension des devoirs et des droits des citoyens.

La matière même des programmes de certains enseignements contribuera à préciser le sens du devoir critique. Les parties communes de ces programmes donneront à tous les jeunes Français une documentation sur les grands faits historiques, économiques, sociaux, susceptibles de leur permettre ultérieurement une meilleure compréhension des événements contemporains et un choix personnel éclairé de leur position idéologique et de leur action civique.

L'exposé et la discussion des événements et des problèmes dans leur actualité ne seront pas exclus de l'école. Des clubs d'étude et de discussion enseigneront aux jeunes la valeur et les difficultés d'une recherche sincère de la vérité, le respect de la liberté de pensée et d'expression pour tous.

Enfin, l'heure obligatoire d'hygiène, dans la dernière année de scolarité du premier degré, comportera des notions d'éducation sexuelle et donnera aux jeunes gens et aux jeunes filles un sens plus exact de leurs devoirs et de leurs responsabilités envers l'humanité à venir. Cependant, l'éducation morale et civique n'aura sa pleine efficacité que si l'influence de l'enseignement proprement dit se complète par l'entraînement à l'action. Le respect de la personne et des droits d'autrui, le sens de l'intérêt général, le consentement à la règle, l'esprit d'initiative, le goût des responsabilités ne se peuvent acquérir que par la pratique de la vie sociale. L'école offre aux enfants et aux adolescents une société à leur mesure, où ils vivent au milieu de leurs pairs. Elle devra donc s'organiser pour leur permettre de multiplier leurs expériences, en leur donnant une part de plus en plus grande de liberté et de responsabilité, dans le travail de classe comme dans les occupations de loisir.

La plupart des services scolaires doivent être pris en charge par les élèves. La mise en ordre de la classe, du matériel scolaire, des bibliothèques seront confiés à des élèves volontaires ou désignés par leurs camarades, responsables devant l'opinion de la bonne exécution du travail. Le rôle du maître sera d'appeler éventuellement l'attention collective sur les injustices dans la répartition des charges. Le sens de la solidarité, à l'intérieur du groupe, prendra toute la force d'une expérience quotidiennement vécue.

L'organisation de coopératives scolaires donnera aux enfants et aux adolescents l'expérience des responsabilités, le sentiment de l'importance des fonctions sociales et de la haute valeur de la conscience dans le travail. C'est par les élèves eux-mêmes que la coopérative doit être gérée. La 
discussion et le choix des travaux à entreprendre, l'attribution à chacun de sa part dans la tâche commune, la valeur des aptitudes, l'acceptation de besognes humbles mais utiles, le sacrifice des préférences personnelles, le renoncement aux satisfactions égoïstes de la vanité pour la joie des réussites collectives, l'harmonisation volontaire des activités individuelles à celles du groupe constitueront une première et utile expérience de la participation à la vie dans une société démocratique. Les travaux et les fêtes organisés par les jeunes coopérateurs et sous leur responsabilité doivent normalement aboutir à doter la collectivité scolaire de fonds dont ils auront la gestion. Ils expérimenteront ainsi l'importance pour le bien commun de l'intelligence et de la probité des gestionnaires et de l'acceptation des décisions collectives ayant force de loi. Dans le cadre des activités coopératives, ou indépendamment d'elles, l'école doit habituer les futurs citoyens à participer à des services sociaux. Par exemple, l'aide aux enfants, aux infirmes, aux vieillards, non par des dons d'argent, mais par la contribution bénévole aux travaux de la vie quotidienne : participation aux besognes ménagères, courses, distractions, etc. De même et particulièrement dans les milieux ruraux, des travaux d'intérêt général peuvent être exécutés par l'équipe scolaire : remise en état de chemins, de clôtures, nettoyages, déblaiements, participation aux grands travaux saisonniers, etc. Ces activités désintéressées seront un moyen efficace d'enseigner aux jeunes à sortir d'eux-mêmes. Ils se prépareront ainsi au devoir commun du service social volontaire qui intègre l'individu par son action et ses sentiments à des groupes humains de plus en plus étendus.

Toutes ces activités scolaires et sociales, ainsi que les jeux, les sports, les occupations éducatives des loisirs, si importantes dans la vie des internats, concourront à donner aux adolescents le sentiment de leur responsabilité sociale, sous deux aspects répondant à la hiérarchie des tâches et des fonctions : responsabilité du dirigeant, responsabilité de l'exécutant. Chaque citoyen, en régime démocratique, est placé dans la vie civique et professionnelle en face de cette double responsabilité. Il sera donc nécessaire que les activités scolaires s'organisent de telle sorte que tous aient alternativement des responsabilités de direction et d'exécution développant conjointement l'initiative, la décision, l'intégration volontaire à une activité réglée et collective, la conscience scrupuleuse dans l'accomplissement des plus modestes tâches. Il importe en effet d'éviter de cultiver en certains l'absolutisme du chef prédestiné et en d'autres l'habitude paresseuse d'une aveugle soumission.

L'organisation des vacances et des loisirs permettra, dans des conditions différentes de celles de la vie scolaire, d'affirmer les qualités acquises. Les colonies enfantines doivent avoir une discipline extrêmement libérale et donner aux enfants l'occasion d'une expérience de vie collective où les interventions adultes sont réduites au minimum et s'exercent avec discrétion. Pour les adolescents, de véritables expériences de "républiques démocratiques" doivent être organisées. Les rassemblements de jeunesse, dans des cités provisoires dont les jeunes assument toute l'organisation et l'administration, sont une excellente préparation à la vie du citoyen. Ils donnent à chacun la possibilité de mesurer les difficultés et les charmes de la liberté individuelle au sein d'une collectivité démocratique.

Le rôle des maîtres dans cette éducation sociale et civique par l'action est important mais discret. Il leur appartient de créer les conditions et l'atmosphère favorables. C'est à eux de doser avec sagesse la part de liberté progressivement étendue qui aboutira, aux $2^{\mathrm{e}}$ et $3^{\mathrm{e}}$ cycles, au régime de l'auto-discipline. Dans toutes les activités collectives libres (services scolaires, coopératives, équipes organisées pour le travail ou pour le jeu), leur rôle est celui d'un témoin et d'un conseiller. Ils n'imposent pas leurs solutions, mais ils aident, ils suggèrent, ils proposent, ils 
s'efforcent de faire reconnaître les erreurs de jugement et de conduite, non en vertu de l'autorité que leur confère leur fonction, mais par le consentement sincère des esprits et des coeurs. A l'enseignement civique est liée l'éducation morale. Ce n'est pas une discipline dont un spécialiste a le monopole. Il appartient à tous les éducateurs de le donner, en toute circonstance, par l'organisation de la vie scolaire, par leur enseignement, par leur exemple. Cependant, au $3^{\mathrm{e}}$ cycle, et surtout dans la classe terminale, un programme portant sur les grands problèmes de la vie civique et morale servira de base à des exposés et des discussions plus méthodiques. Il permettra avant que les adolescents ne quittent l'école, de faire une synthèse des connaissances acquises et de rendre conscients les principes qui règlent la conduite et fondent le jugement moral.

Ainsi, l'éducation intellectuelle et la pratique quotidienne de l'expérience sociale, dans ce " groupe scolaire à structure démocratique auquel l'enfant participe comme futur citoyen », contribueront conjointement à former l'homme et le citoyen, à éclairer l'action par la pensée, à vivifier la pensée par l'action. Et comme l'écrivait Paul Langevin : «Cet apprentissage de la vie sociale, essentiellement laïque, n'engage aucune idéologie, n'exige aucune mystique métaphysique ou religieuse ». L'école, mettant en évidence les éléments communs de nos devoirs d'homme et de citoyen, contribuera efficacement à renforcer le sentiment de la communauté française.

VII

\section{EDUCATION POPULAIRE}

L'éducation populaire n'est pas seulement l'éducation pour tous, c 'est la possibilité pour tous de poursuivre au-delà de l'école et durant toute leur existence le développement de leur culture intellectuelle, esthétique, professionnelle, civique et morale.

Dans des temps où les progrès des sciences et le renouvellement des idées et des manifestations artistiques ne peuvent manquer de s'accélérer toujours davantage, les générations qui se suivent deviendraient vite étrangères entre elles et les plus anciennes étrangères à leur époque, si cette possibilité ne leur était pas donnée.

L'éducation populaire ne doit pas être la simple continuation de l'école avec emploi de méthodes scolaires pour compléter une instruction jugée insuffisante. S'adressant aux adultes elle doit partir de leurs intérêts actuels et utiliser leurs aptitudes d'adultes.

Elle doit être à la fois représentée sur tous les points du territoire et garder le contact avec les institutions et les hommes dont la mission est le progrès de nos connaissances culturelles. Elle exige la collaboration de tous à quelque niveau de l'enseignement qu'ils appartiennent : maîtres répandus dans les campagnes et dans les villes d'une part, maîtres des écoles normales et des universités d'autre part. Cette collaboration sera d'autant plus facile que tous les maîtres seront passés par les écoles normales et les universités.

Les écoles normales et les universités seront des foyers de culture où les maîtres, en contact direct avec les populations, devront trouver l'assistance et les collaborations voulues pour organiser dans leur propre circonscription des séances instructives ou récréatives, des excursions géologiques, botaniques, archéologiques, etc., des expositions et des festivités soit de caractère régional, soit de caractère national ou mondial. Cette énumération n'a rien de limitatif.

La fonction d'éducation populaire est d'importance trop fondamentale pour que les maîtres la remplissent à leurs moments perdus. Elle ne saurait s'ajouter à leurs charges professionnelles déjà lourdes, qui doivent être allégées en proportion. 
Mais il faut aussi envisager que dans les limites par exemple du canton, des maîtres seront entièrement délégués dans cette fonction. Cette délégation ne sera pas une délégation à vie, mais à temps. Elle permettra d'introduire plus de diversité dans l'existence professionnelle des maîtres, dont la monotonie est parfois trouvée rebutante et peut décourager certains au moment de s'y engager. Des stages d'information pourront être organisés pour les candidats à cette fonction.

Si l'armature de l'éducation populaire doit être formée par le personnel enseignent à tous ses degrés, elle devra également s'assurer la collaboration de toutes les organisations, publiques ou privées, dont le but est culturel : associations pour la connaissance du milieu historique ou naturel, pour le développement des arts et de la littérature.

Ainsi l'éducation populaire sera un ferment du progrès intellectuel, technique, esthétique non seulement pour les individus, mais pour la collectivité.

\section{VIII}

\section{DELAIS D'APPLICATION}

C'est à la loi de la réforme scolaire qu'il appartient de fixer les délais dans lesquels ses différentes parties devront être appliquées. En effet, des paliers sont nécessaires. Mais, faute d'en fixer la succession et la durée, ses mesures les plus essentielles risqueraient d'être indéfiniment ajournées.

Trois sortes d'obstacles peuvent être envisagées : $1^{\circ}$ les besoins du pays en main-d'oeuvre auxquels la prolongation de la scolarité pourrait porter préjudice. $2^{\circ}$ L'insuffisance des bâtiments scolaires pour un volume accru d'élèves. $3^{\circ}$ Le nombre déjà trop réduit des maîtres que l'augmentation de la clientèle scolaire et le temps plus long de leur formation d'autre part rendront plus insuffisant encore.

$1^{\circ}$ La scolarité obligatoire sera d'abord portée de 14 à 15 ans, l'année qui s'ajoute devant être, pour commencer, une année pratique préparant à la profession. Elle prendra place dans les établissements du $2^{\mathrm{e}}$ cycle (à la campagne écoles intercommunales). Elle introduira donc dans le second cycle des maîtres de l'enseignement technique qui, dans la suite auront à y diriger les options.

Faute de bâtiments scolaires suffisants, il ne sera pas possible de porter partout à la fois la scolarité de 14 à 15 ans. Néanmoins le regroupement dans les écoles intercommunales de tous les enfants en âge de suivre le second cycle devra s'opérer immédiatement.

Dans cinq ans au plus tard la scolarité devra être portée de 15 à 18 ans. Les établissements qui auront à recevoir les enfants suivant les cas, les écoles d'apprentissage, les écoles professionnelles, les établissements du $3^{\mathrm{e}}$ cycle menant au baccalauréat et à l'enseignement préuniversitaire. Les bâtiments scolaires à prévoir seront donc différents des précédents et les effectifs à recevoir seront la première année d'un tiers, puis la deuxième de deux tiers avant d'être complets.

$2^{\circ}$ Le problème des bâtiments à prévoir est essentiel. Dans le mois qui suivra le vote de la loi, des commissions départementales devront être constituées pour établir la carte scolaire des régions. Elles comprendront des représentants de l'administration universitaire, des représentants des assemblées élues (conseils généraux et municipalités) des représentants du corps enseignent et des grandes organisations économiques. 
Dans le délai maximum de six mois elles devront avoir établi la carte scolaire : répartition des bâtiments existants entre les différents enseignements et projet d'aménagement. Indication des établissements à construire, affectation, localités, importance.

Les problèmes de construction seront réglés en collaboration avec des comités techniques.

Le programme de constructions scolaires doit être inclus dans le plan Monnet parmi les sections prioritaires car l'équipement du pays en travailleurs qualifiés (bons apprentis, bons techniciens, bons chercheurs) est celui qui importe le plus à son relèvement.

Les délais d'exécution de constructions scolaires s'inscriront ainsi dans les délais d'exécution du plan.

$3^{\circ}$ Le recrutement des maîtres qui tend à se tarir devra être considérablement amplifié. Sinon ce n'est pas seulement le sens de la réforme qui serait compromis mais c'est l'existence de l'Université elle-même, dans son état actuel. L'enseignement sera de plus en plus déserté pour d'autres situations plus lucratives, s'il ne donne pas à ceux qui veulent s'y consacrer des avantages en rapport avec les études qui sont exigées d'eux. La revalorisation équitable de la fonction enseignante est d'autant plus urgente que la formation des maitres doit être encore élevée d'un niveau.

Le passage de tous les maîtres par l'Université exigera une augmentation de cadres qualifiés dans l'enseignement supérieur. Aussi peut-on prévoir qu'un délai de cinq ans sera nécessaire avant que cette mesure soit complètement appliquée. Durant la période intermédiaire, des maîtres en proportion variable pourront être appelés à enseigner dès la fin de leurs années préuniversitaires (Ecoles normales). Ils pourront être admis dans la suite à faire des stages compensateurs.

\section{APPENDICE}

\section{1 - PROJET D'ORGANISATION DE L'ENSEIGNEMENT DANS LES REGIONS RURALES}

\section{A - UNITE DE L'ENSEIGNEMENT TECHNIQUE}

L'enseignement technique rural destiné aux enfants dont les deux premières années d'orientation n'ont pas déterminé la sélection en vue d'études de longue durée (au-delà de 18 ans) doit préparer :

a) Aux diverses professions paysannes et agricoles : paysan polyvalent, chef d'exploitation, spécialiste (vacher, viticulteur, maraîcher, etc.), vétérinaire, zootechnicien, généticien, entrepreneur de travaux de génie rural ;

b) Aux professions de l'artisanat et du commerce rural et cela en tenant compte de l'introduction croissante de techniques industrielles à la campagne (mécanique, électricité, usage du froid, pasteurisations, etc.) et de l'impossibilité, dans les pays de petite propriété ou de petite exploitation, d'absorber dans la profession agricole tous les enfants d'une famille de trois ou de plus de trois enfants.

Il ne saurait donc être exclusivement agricole. Pour que la répartition des élèves entre les différentes branches s'effectue dans les meilleures conditions, il est indispensable que tout cet enseignement technique soit donné dans des écoles à plusieurs spécialités, disposant d'annexes 
d'application et dépendant du Ministère de l'Education nationale au point de vue administratif et financier.

$2^{\circ}$ L'enseignement technique recrute ses maîtres dans les écoles préparatoires dépendant du Ministère de l'Education nationale et ses moniteurs dans les diverses professions, sous réserve d'un stage probatoire, dans les cadres de l'Education nationale, mettant à l'épreuve leurs qualités pédagogiques. Des personnalités des divers Ministères et des diverses professions peuvent être appelées à enseigner dans les écoles préparant le personnel de l'enseignement technique.

$3^{\circ} \mathrm{Il}$ recourt, pour les dotations en matériel, aux divers Ministères économiques (en l'espèce, au Ministère de l'Agriculture et aux grandes collectivités coopératives, C. G. A., etc...)

\section{B - ORGANISATION DE L'ENSEIGNEMENT DU 1er CYCLE DU 2e DEGRE A LA CAMPAGNE}

$1^{\circ}$ Ecoles intercommunales. - L'enseignement du second degré au niveau des années d'orientation (11-15 ans, tout au moins 11-13 ans) doit être donné par plusieurs maîtres et offrir un jeu d'options adapté à la vie rurale qui ne peut être assuré que dans des centres scolaires plus importants que la plupart des communes rurales françaises.

Cependant, pour ne pas astreindre les jeunes ruraux à des déplacements trop longs, il paraît sage de ne pas se limiter à l'établissement d'écoles cantonales. La formule d'une école intercommunale paraît, à tous égards, préférable, dans la généralité des cas.

La création des écoles intercommunales doit s'accompagner d'une organisation sérieuse du transport et du séjour journalier des enfants dans la localité choisie comme lieu de ralliement (cantine, loisirs, etc ...)

$2^{\circ}$ Fréquentation scolaire. - L'école intercommunale dispensera la scolarité annuelle complète, l'enseignement des deux premières années d'orientation.

A 13 ans, on admettra, au moins pour une période d'attente de quelques années, que l'essentiel de l'orientation scolaire soit fait. Pour les enfants se destinant à l'enseignement pratique (le plus grand nombre) la fréquentation de l'école se bornera à la période d'hiver (du $1^{\text {er }}$ novembre au 31 mars), pendant laquelle sera réparti un total d'heures obligatoires comportant, pour parties égales, un enseignement général et un enseignement optionnel qui, à cet âge, ne saurait revêtir l'aspect d'un enseignement professionnel proprement dit.

Les enfants ne destinant à l'enseignement professionnel, carrières de cadres agricoles, chefs d'exploitation, d'institutions collectives, spécialistes d'agronomie, etc., cadres industriels et administratifs, suivront, au contraire, un enseignement de pleine fréquentation annuelle jusqu'à 15 ans, date de leur entrée dans une école professionnelle.

Il est à prévoir que la proportion des élèves ayant intérêt à suivre la formation professionnelle s'accroîtra progressivement, en fonction de l'amélioration des méthodes d'exploitation agricole.

$3^{\circ}$ Organisation de l'enseignement. - Jusqu'à 11 ans, l'enseignement du premier degré demeure à peu près identique dans toutes les écoles, urbaines ou rurales, au moins dans son objet. Mais il est recommandable d'éveiller la notion de solidarité entre l'école conçue comme communauté locale et le milieu. 
De 11 à 15 ans (premier cycle du second degré), développement des options dans le cadre du milieu rural, par conséquent avec une très grande souplesse suivant les régions. Au maximum d'ouverture de l'éventail des options, on peut envisager le problème suivant :

a) Option agricole, dans certaines régions, botanique, pédologique, culturale, dans d'autres, zoologique, forestière, etc, avec, à partir de 13 ans, initiation à des travaux pratiques, sans prétention d'apprentissage proprement dit, destinés seulement à vérifier des aptitudes et éveiller des goûts.

b) Option artisanale, également variable suivant les régions, comportant partout, cependant, une branche "bâtiment" et une branche "mécanique".

c) Option industrielle agricole, initiant aux métiers des industries agricoles locales : fromageries, sucreries, fabrication des conserves, etc.

d) Option administrative et sociale, initiant aux diverses formes de la vie sociale : droit élémentaire pratique, hygiène sociale, commerce, administration, problème de l'enfance et de l'éducation.

Les sujets nettement qualifiés pour des carrières urbaines, ou pour des carrières de cadres supérieurs exigeant des études de longue durée, seront dirigés, à 12 ou 13 ans vers les centres scolaires équipés pour préparer soit aux études théoriques de longue durée, soit à l'enseignement professionnel industriel.

$4^{\circ}$ Maîtres . - Pour l'enseignement général, des instituteurs, choisis parmi ceux qui ont le plus d'aptitudes pour dispenser l'enseignement à des enfants de cet âge, éventuellement professeurs de cours complémentaires.

Pour l'enseignement des matières à options, des professeurs ayant reçu une formation spéciale par voie de stage dans des écoles professionnelles ou dans des laboratoires d'Universités, des stations d'essai ou des ateliers-écoles, etc ...

Toutefois, pour faciliter le recrutement des maîtres d'options agricoles, tous les instituteurs ruraux devront recevoir une formation élémentaire agricole.

$5^{\circ}$ Prévision des variations du marché du travail. - Prévoir :

a) La résorption des excédents d'enfants qui ne trouveront plus de débouché dans l'agriculture du fait de l'accroissement de la superficie moyenne exploitée par un ménage (seul moyen d'équilibrer le niveau de vie de la famille rurale ou celui de la famille ouvrière et de la rationalisation du travail de la terre ;

b) Les besoins de nouvelles professions techniques : mécaniciens, conducteurs et réparateurs de machines, service d'entretien du petit matériel, électriciens, chimistes, biologistes ;

c) Les besoins du personnel qualifié, en fonction de l'équipement social et commercial des campagnes : personnel sanitaire, personnel des coopératives, personnel administratif, personnel des services agricoles de base, conseillers agricoles, techniciens divers, etc...

Il s'agit de porter à la connaissance des enfants la réalité de chacune des professions appelées à se développer à la campagne pour éveiller des vocations et orienter des efforts.

d) Propagande en faveur de l'enseignement à la campagne. Les parents n'envoient souvent pas leurs enfants à l'école, parce qu'ils pensent que c'est inutile. Cette impression est justifiée parfois par l'absence totale de liaison entre le travail fait à l'école et la vie concrète du pays. Mais elle est aussi le résultat de l'ignorance par beaucoup de paysans de l'intérêt des valeurs culturelles et même de la formation technique. 
Nécessité de traiter des problèmes de l'école rurale dans tous les journaux régionaux et professionnels agricoles, vulgariser les expériences existantes par le film, la radio, etc ... Insister sur l'idée qu'un paysan instruit et formé techniquement gagne plus d'argent que celui qui a reçu seulement une formation empirique dans le cadre de l'entreprise familiale.

Vulgariser par brochures avec illustrations, photographies, ,etc ...

\section{C - ENSEIGNEMENT PRATIQUE ET ENSEIGNEMENT PROFESSIONNEL À LA CAMPAGNE (15 à 18 ANS)}

\section{SERIE PROFESSIONNELLE}

$1^{\circ}$ Objet. - Formation des cadres techniques ruraux : conseillers agricoles, zootechniques, vétérinaires, directeurs et gérants des institutions, collectivités, syndicats, coopératives, caisses d'assurances, service du cadastre et des remboursements, dirigeants des services administratifs ruraux, directeurs des services d'équipements mécaniques, électriques, des transports, etc., dirigeants des industries de transformation des produits agricoles.

\section{$2^{\circ}$ Organisation.}

Ecole d'Agriculture de pleine fréquentation comportant :

une section générale ;

une section zootechnique et vétérinaire ;

une section administrative.

Ecole normale préparant à toutes les fonctions enseignantes ;

Ecole professionnelle dont l'orientation précise sera déterminée :

a) Par la nature des industries existent dans la région ou que l'on souhaite y introduire ;

b) Par les besoins de l'équipement régional.

\section{II - SERIE PRATIQUE}

$1^{\circ}$ Objet. - Formation de bons travailleurs dans les différents domaines de l'activité régionale.

$2^{\circ}$ Organisation. - Ecole pratique agricole : Ferme-Ecole de fréquentation saisonnière, associant l'enseignement général à la formation pratique. Stages contrôlés chez l'exploitant, et auprès des institutions collectives. Comporte initiation au travail de la terre et à l'entretien de tout matériel agricole.

Ecole pratique d'artisanat rural, centre de formation professionnelle, sections bâtiment, forge, serrurerie, électricité, mécanique, automobile, bois et cuir.

Ecole ménagère, hôtelière, etc., spécialement pour les jeunes filles, avec stages contrôlés au domicile familial et dans les entreprises privées ou collectives.

Ecole pratique commerciale et administrative, permettant de recruter dans de bonnes conditions :- employiés de chemin de fer, facteurs, gendarmes, de former des commerçants de village, etc ...

Enseignement professionnel. 
- Localisation : chef-lieu de département ou chef-lieu d'arrondissement, avec internat gratuit et présalaire.

- Personnel. : Personnel de l'enseignement technique, une partie peut-être formée, notamment pour la branche enseignement agricole, à partir des instituteurs ruraux. Ce personnel est assisté de moniteurs.

Enseignement pratique.

- Localisation : peut et doit être beaucoup plus dispersé que l'enseignement professionnel (nombre d'élèves bien plus considérable). Ecoles pratiques d'artisanat rural et centres de formation professionnelle, école pratique commerciale et administrative au chef-lieu de canton. Fermes-écoles intercommunales, ou même, dans les régions de fort groupement rural, communales.

- Personnel : les instituteurs représentant la base principale de recrutement du personnel, enseignement général et enseignement théorique de la profession (après stage approprié), direction des centres au point de vue administratif et surtout moral. Les instituteurs détachés dans ces fonctions recevront une indemnité sanctionnant leur qualification. Ils seront assistés par des moniteurs.

- Besoins : nécessité de donner satisfaction immédiate au monde rural qui veut dès maintenant que la jeunesse reçoive le complément d'éducation et d'instruction nécessaire aux futurs agriculteurs et aux futurs artisans, de tous ordres, qui habitent la campagne.

- Organisation : il faut que cet enseignement agricole et ménager post-scolaire soit donné aux heures normales de travail des jeunes gens et des jeunes filles, c'est-à-dire pendant la journée. Les séances du soir ne pourront qu'être exceptionnelles.

Les déplacements des jeunes gens qu'exigera cet enseignement ne peuvent avoir lieu au cours de la nuit ou au cours des veillées. Les familles, dans la plupart des cas, se refuseraient à laisser ainsi la jeunesse se rendre au cours.

Il faut que les cours soient donnés en des lieux qui ne soient point trop éloignés de la résidence des adolescents. Le projet qui consiste à faire donner cet enseignement post-scolaire aux chefslieux de canton ne donnera pas de résultat quant à la fréquentation.

Il faut également que l'équipement de ces centres en cantines et que l'organisation des transports des élèves soient assurés.

Il est donc recommandable d'installer les centres d'enseignement post-scolaire agricole dans des écoles intercommunales qui seront pourvues ultérieurement d'un enseignement régulier du premier cycle du second degré et de fermes-écoles : voir § B et C.

$3^{\circ}$ Nature de l'enseignement. - Le caractère de cet enseignement comporte forcément des notions pratiques et des notions théoriques. Les notions pratiques pourront être données verbalement, mais, toutes les fois qu'il sera possible, il faudra que jeunes gens et jeunes filles se livrent effectivement à des travaux pratiques. Un enseignement général accompagnera cet enseignement théorico-pratique.

$4^{\circ}$ Personnel. - Instituteurs ruraux issus de milieux d'agriculteurs ayant suivi l'enseignement agricole à l'Ecole Normale, âgés de 30 ans au moins, volontaires et subissant un stage de 3 à 6 mois dans une école d'agriculture ou dans. un centre universitaire d'applications agricoles (Toulouse). Ces maîtres seront remplacés dans leurs fonctions par des intérimaires actuellement sans emploi. Ils recevront une indemnité de fonction. 
$5^{\circ}$ Fréquentation. - Une à deux journées par semaine, les maîtres desservant chacun trois centres intercommunaux.

Cet enseignement sera essentiellement un enseignement de saison d'hiver. Cependant, certains travaux d'application à la ferme-école devront avoir lieu pendant la saison des travaux agricoles, seul moyen de donner une valeur concrète à un enseignement destiné au travail à effectuer pendant cette saison même.

\section{II - VOEUX EMIS PAR LA COMMISSION AU COURS DE SES SEANCES}

\section{Budget de l'Education Nationale}

La commission ministérielle d'étude pour la réforme de l'enseignement, unanime dès le début de ses travaux pour affirmer la nécessité d'une organisation permettent à tous les enfants de développer pleinement leurs aptitudes, de manière que la place de chacun dans la société soit déterminée par sa seule valeur personnelle, tient à appeler dès maintenant l'attention des Pouvoirs publics sur les mesures qui sont à prendre immédiatement pour assurer l'application de ces principes, et sur l'importance considérable de l'effort financier qu'elle implique. Gratuité de l'enseignement public à tous les degrés.

Octroi de larges allocations familiales et bourses permettant de réaliser sur le plan matériel les qualités de tous devant l'enseignement.

Mise à exécution aussi rapide que possible d'un large programme de constructions modernes et universitaires.

Et. enfin, remise en l'honneur et revalorisation de la fonction d'enseignement à tous les degrés, afin de donner aux membres du personnel l'indispensable situation morale et l'indépendance qu'exige l'importance de leur rôle social, autant que pour leur assurer un recrutement qualifié, surtout dans une période où leur nombre doit être considérablement augmenté.

Les prévisions faites pour le budget de l'Education nationale dans les divers pays étrangers, en Grande-Bretagne, en particulier, montrent combien la situation correspondante en France est actuellement indigne de notre grand pays.

\section{La Commission,}

Considérant que les crédits affectés à l'Education Nationale sont nettement insuffisants, Que la préparation de la Réforme de l'enseignement et que l'accroissement des effectifs scolaires nécessitent une augmentation substantielle des dotations des services de l'Enseignement, Emet le voeu que des dispositions législatives et budgétaires soient prises le plus rapidement possible pour tenir compte de cette situation, Que la réduction de $7 \%$ des dépenses des administrations de l'Etat ne soit pas appliquée aux dépenses de l'Education Nationale, Que l'Education Nationale participe au Conseil du Plan et mette au point un plan d'équipement scolaire et universitaire

Que soit créé un Centre National d'outillage des laboratoires et établissements des Premier et Deuxième degrés. 


\section{Créations d'emplois}

La Commission,

Considérant l'augmentation du nombre des naissances qui provoque dès à présent un

accroissement des effectifs des écoles maternelles et lui permet de prévoir un accroissement de $50 \%$ des effectifs actuels dans l'enseignement obligatoire dès 1951,

Considérant que la mise en application progressive de la Réforme de l'enseignement entraînera

dès 1948 le maintien à l'école pour une année supplémentaire de 400000 enfants,

Considérant qu'il est du devoir de l'Etat de permettre à tous les enfants de France de fréquenter

l'école publique,

Demande que soient dès à présent ordonnées les création $\mathrm{s}$ d'emplois indispensables pour

répondre aux besoins ; que les effectifs des Ecoles Normales ne soient pas cette année

déterminés en fonction du nombre des retraites, mais que soient prises toutes dispositions pour assurer la formation professionnelle des nouveaux maîtres (augmentation des effectifs des Ecoles Normales, passage par l'Ecole Normale du plus grand nombre possible de suppléants, organisation de stages obligatoires de formation professionnelle pour les futurs maîtres suppléants et intérimaires).

\section{Locaux scolaires}

La réorganisation de la carte scolaire, les besoins nouveaux créés par les changements à intervenir dans les programmes et les méthodes de l'enseignement des 3 cycles du $1^{\text {er }}$ degré, entrainant des modifications dans la conception des bâtiments- scolaires,

La Commission émet le vœu :

Qu'une Commission interministérielle restreinte soit immédiatement constituée, comprenant des techniciens de la construction et de l'enseignement pour déterminer le nombre et l'affectation des locaux jugés indispensables et pour établir le type des constructions nouvelles.

Les dispositions arrêtées devront être portées à la connaissance de MM. les Inspecteurs d'Académie, de MM. les Préfets et Maires dès le $1^{\text {er }}$ octobre 1947.

\section{Formation des maîtres}

La Commission émet le voeu que tout maître, enseignant dans un établissement français doit posséder les titres universitaires et présenter les garanties professionnelles prévus par le projet de Réforme de l'Enseignement.

L'organisation scolaire prévue par la Réforme doit être appliquée dans tous les établissements d'enseignement.

La Commission considérant la nécessité de plus en plus urgente, pour les professeurs des différents ordres, de se tenir au courant des progrès de la science ou de la pédagogie et d'être en mesure d'y participer, en vue même de la valeur et de l'efficacité de leur enseignement, émet le voeu que des périodes de travail libre (année sabbatique, détachements temporaires à la recherche, etc ...) soient assurés à chacun d'eux dans des conditions à préciser statutairement.

\section{Réforme de l'orthographe}

La Commission considérant la charge présente qu'une orthographe irrationnelle et reposant souvent sur des fondements linguistiques peu sûrs fait peser sur notre enseignement et sur la diffusion - très menacée - du français à l'étranger, après avoir discuté et approuvé dans son ensemble le projet de réforme de l'orthographe dont elle avait confié l'élaboration à MM. Hubert Pernot et Charles Bruneau, en recommande l'adoption comme hautement désirable dans les plus 
brefs délais et estime que la réforme ne pourra être effectivement réalisée que si elle devient la règle et est imposée à ce titre à toutes les publications. 